San Jose State University

SJSU ScholarWorks

Master's Theses

Master's Theses and Graduate Research

Fall 2013

\title{
Correlation of Surface Enhanced Raman Spectroscopy and Nanoparticle Aggregation with Rhodamine 6G
}

Christopher Aaron Hoff

San Jose State University

Follow this and additional works at: https://scholarworks.sjsu.edu/etd_theses

\section{Recommended Citation}

Hoff, Christopher Aaron, "Correlation of Surface Enhanced Raman Spectroscopy and Nanoparticle Aggregation with Rhodamine 6G" (2013). Master's Theses. 4390.

DOI: https://doi.org/10.31979/etd.mqys-85aa

https://scholarworks.sjsu.edu/etd_theses/4390

This Thesis is brought to you for free and open access by the Master's Theses and Graduate Research at SJSU ScholarWorks. It has been accepted for inclusion in Master's Theses by an authorized administrator of SJSU ScholarWorks. For more information, please contact scholarworks@sjsu.edu. 


\title{
CORRELATION OF SURFACE ENHANCED RAMAN SPECTROSCOPY AND NANOPARTICLE AGGREGATION WITH RHODAMINE 6G
}

\author{
A Thesis \\ Presented to
}

The Faculty of the Department of Chemistry

San José State University

In Partial Fulfillment

of the Requirement for the Degree

Master of Science

by

Christopher A. Hoff

December 2013 
(C) 2013

Christopher A. Hoff

ALL RIGHTS RESERVED 
The Designated Thesis Committee Approves the Thesis Titled

\section{CORRELATION OF SURFACE ENHANCED RAMAN SPECTROSCOPY AND NANOPARTICLE AGGREGATION WITH RHODAMINE 6G \\ by}

Christopher A. Hoff

APPROVED FOR THE DEPARTMENT OF CHEMISTRY

SAN JOSÉ STATE UNIVERSITY

DECEMBER 2013

Dr. Roger Terrill Department of Chemistry

Dr. Joseph Pesek Department of Chemistry

Dr. Marc d'Alarcao Department of Chemistry 


\title{
ABSTRACT \\ CORRELATION OF SURFACE ENHANCED RAMAN SPECTROSCOPY AND NANOPARTICLE AGGREGATION WITH RHODAMINE 6G
}

\author{
by Christopher A. Hoff
}

Surface enhanced Raman spectroscopy (SERS) has fascinated the analytical chemistry field for decades. The SERS phenomenon has progressively leveraged the inherently insensitive Raman phenomenon from a novelty vibrational spectroscopy method into one capable of single molecule detection, with attendant molecular level selectivity and information. Yet, even after 40 years since its discovery, the core mechanism behind this phenomenon is still debated. This thesis presents results from a series of photometric titrations wherein solutions of $30 \mathrm{~nm} \mathrm{Au} @ \mathrm{Ag}$ nanoparticles (NPs) were titrated with rhodamine $6 \mathrm{G}$ (R6G), spanning five orders of magnitude in R6G concentration, and which elucidate the conditions required for the onset of SERS by R6G in this system. The experiments illustrated the correlation between the Raman response and the plasmonic (via UV-Vis spectroscopy) properties of the nanoparticle solutions. It was found that the onset of R6G SERS was related much more closely to the aggregation of the nanoparticles in solution than to their R6G adsorbed surface coverage. However, triggering aggregation with sodium chloride appeared to enhance SERS by an independent mechanism, which is operative only at low, i.e., $[\mathrm{NaCl}]>100 \mathrm{mM}$ concentration. 


\section{ACKNOWLEDGMENTS}

I would like to express my gratitude to Dr. Terrill for all the time and effort he has dedicated to me through the process, and for his kind nature of entertaining all my preposterous theories. The first and most memorable lesson he bestowed upon me was the demonstration of the carnage that follows when torsional force is applied to a Schlenk line. I would also like to thank Dr. d'Alarcao for being part of my thesis committee and instilling an everlasting fear of $3 \times 5$ cards with my name on it. Dr. Pesek was the first person that welcomed me into the master's program. I am honored to have all three professors on my committee and to give validation to the work I have done.

I also have to express my appreciation to all my peers that have accompanied me through this process. They say that the strongest bonds are forged in the trenches. I will always remember the conversations in the halls of Duncan and the tables in McDonalds.

Lastly, I have to mention the support that I received from the friends outside of chemistry and Family. I will cherish the glossy eyed looks I received when rehearsing my prelim and final seminar.

Seriously, I would like to say thank you to everyone through this process. I did not accomplish this on my own. 


\section{Table of Contents}

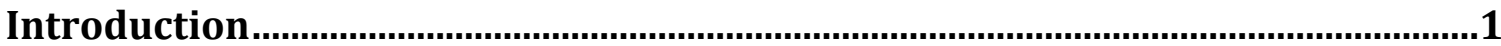

Raman Scattering Spectroscopy

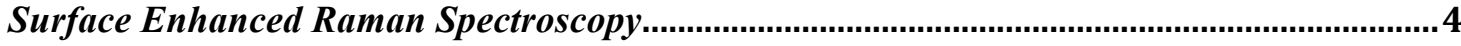

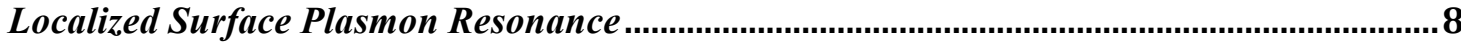

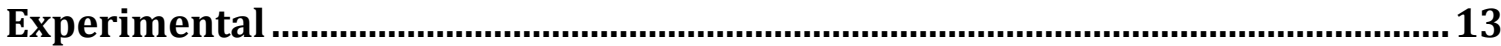

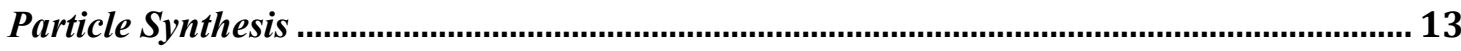

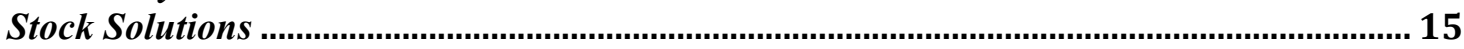

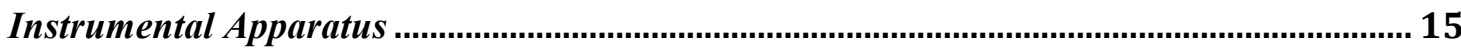

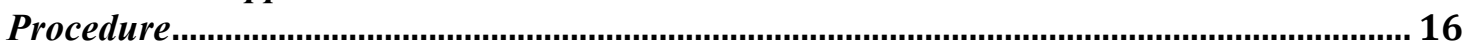

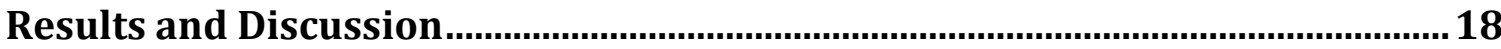

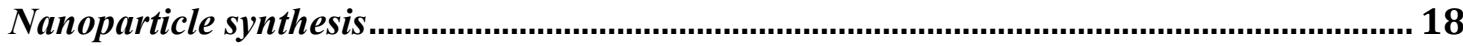

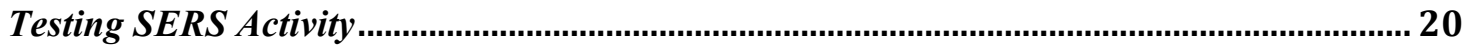

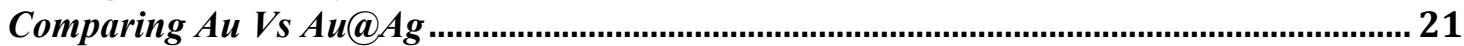

Nanoparticle Titration with R6G

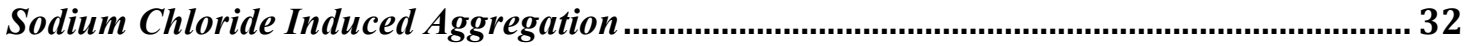

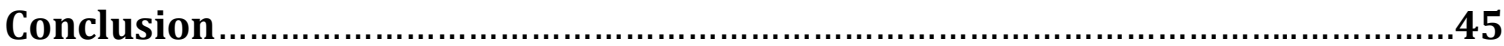

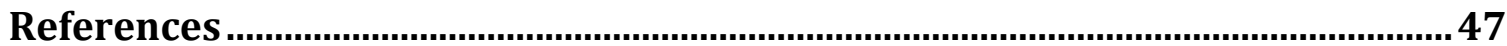




\section{List of Figures}

Figure 1: Raleigh and Raman scattering .................................................................................... 3

Figure 2: The electric field of the Electromagnetic radiation induces a polarization of the conduction electrons in the metal nanoparticles......................... 11

Figure 3: $\mathrm{Au}, \mathrm{Ag}$ and $\mathrm{Au} @ \mathrm{Ag}$ nanoparticles absorbance spectra ...................................... 20

Figure 4: Different Raman spectra of 2.6 $\mu \mathrm{M}$ R6G in presence of $\mathrm{Au} @ \mathrm{Ag}$ nanoparticles and without nanoparticles...................................................................... 21

Figure 5: SERS of R6G of the same concentration differing only in the composition of the nanoparticle................................................................................... 22

Figure 6: Raman spectra recorded during titration of $\mathrm{Au} @ \mathrm{Ag}$ with R6G......................... 23

Figure 7: Raman spectra acquired as R6G is titrated into pure water ................................ 24

Figure 8: Vis-NIR spectra acquired as Au@Ag is titrated with R6G................................ 24

Figure 9: Compiled SERS and UV-Vis Spectrums of R6G titrated into Au@Ag nanoparticle solution .............................................................................................................. 25

Figure 10: Illustration of the formation of interparticle "hot spots" when the light electrical field is parallel to interparticle axis ............................................................... 27

Figure 11: Nanoparticle aggregation spectra in the UV-Vis with R6G absorption spectrum removed ............................................................................................................ 29

Figure 12: 4-Mercaptopyridine titrated into an Au nanoparticle solution .......................... 30

Figure 13: Transverse dipolar mode that is created in the nanoparticle dimers when light electric field is orthogonal to the interparticle axis.

Figure 14: (a) is a diagram that illustrates both the order of addition and the final concentrations of $\mathrm{R} 6 \mathrm{G}$ and $\mathrm{NaCl}$ that are spiked into the $\mathrm{Au} @ \mathrm{Ag}$ solutions. Plots of Raman spectra (b) and visible extinction spectra (c) of each endpoint $(\bullet)$

Figure 15: DVLO simulations for Ag nanoparticles coated with citrate ions with a radius of $20 \mathrm{~nm}$ at $25^{\circ} \mathrm{C}$ at $10 \mathrm{mM}$ (a), $100 \mathrm{mM}$ (b) and $150 \mathrm{mM}$ (c)

Figure 16: The SERS spectra of $0.125 \mathrm{mM} \mathrm{R6G}$ before (black) and after (purple) the $\mathrm{NaCl}$ concentration was increased to $100 \mathrm{mM}$ from $10 \mathrm{mM}$.

Figure 17: Shows that the SERS spectrum of R6G has nearly identical Raman shifts as the SERS spectrum of R6G with the addition of $\mathrm{NaCl}$ 
Figure 18: Raman spectrum of $\mathrm{Au} @ \mathrm{Ag}$ (red) and following addition of $150 \mathrm{mM}$ $\mathrm{NaCl}$ (green).................................................................................................................... 42

Figure 19: Chemical and 3D representation of R6G............................................................43 


\section{List of Tables}

Table 1: Universally applied spike titration series used for all analytes .......................... 18

Table 2: Legend of details pertaining to Figure 14 Vis-NIR and Raman spectra............ 35

Scheme 1: Schematic that demonstrates the effects of addition of $\mathrm{NaCl}$ into the

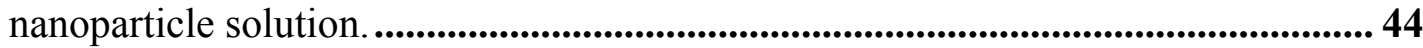




\section{Introduction}

\section{Raman Scattering Spectroscopy}

In 1928, during the inaugural address at the South Indian Science Association in Bangalore, Sir Chandrasekhara Venkata Raman delivered a speech titled, "A new Radiation." The new type of radiation he was referring to would later be called Raman scattering in his honor. Raman scattering is the inelastic scattering of a photon, (Figure 1). When a molecule is irradiated with a monochromatic beam with the energy $h v_{\mathrm{ex}}$, the molecule can be excited into one of the infinite virtual states that are between the ground state and the lowest lying (first) electronically excited state. When the molecule returns to the ground state, a photon may be reemitted. If the photon emitted is of the same frequency, the scattering is termed elastic and this phenomenon is called Rayleigh scattering in honor of the classical description still used to quantify this phenomenon

today. $^{2}$ In contrast, Raman scattering is inelastic because the photon reemitted has a different frequency (Figure 1), described as Stokes scattering if the new photon is at a lower frequency than the original and anti-Stokes scattering if it is at a higher frequency higher. ${ }^{3}$ When viewed as a scattering spectrum of intensity versus light frequency, the Stokes and Anti-Stokes peaks appear at symmetrically higher and lower frequencies relative to the much more intense Rayleigh scattering peak. The relative intensities of the Stokes and anti-Stokes peaks depend on temperature, but Stokes are normally the more intense of the two owing to the relatively larger population of vibrational ground states as depicted in Figure 1. 
Perhaps the most appealing aspect of Raman spectroscopy is that it presents the detailed, unique, and informative vibrational spectrum of the molecule under study. This occurs because Raman peaks appear for every Raman active vibrational mode at the laser excitation minus the vibrational frequency. Thus, Raman provides information equivalent to that which can be derived from Infrared spectroscopy (IR) but in the much more experimentally convenient optical frequency regime. Optical excitation frequencies can present a large advantage in terms of sample preparation, unlike for infrared spectroscopy, Raman may be done in aqueous solvents (owing to the extremely low Raman cross section of water) and using conventional lens and sample holding materials such as borosilicate glasses. ${ }^{2}$ Normal Raman spectroscopy would thus seem to be a natural choice replacement for infrared spectroscopy, but it does suffer from a major constraint - Raman scattering is, fundamentally, an exceptionally weak phenomenon, only about one ten-millionth as intense as the already weak Rayleigh scattering from molecules. ${ }^{4}$ For this reason, Raman spectroscopy has been slow to evolve into a generally useful analytical tool and in the past has required long integration times. But with the advent of a complement of methodologies ranging from intense semiconductor lasers, confocal backscattering collection geometries, holographic notch filtering to remove interference from Rayleigh photons, and sensitive integrating CCD spectrographic detection, Raman spectra of many compounds can now be acquired quickly and conveniently with relatively inexpensive (e.g., $<\$ 20,000)$ instrumentation. 


\section{Stokes Anti-Stokes}

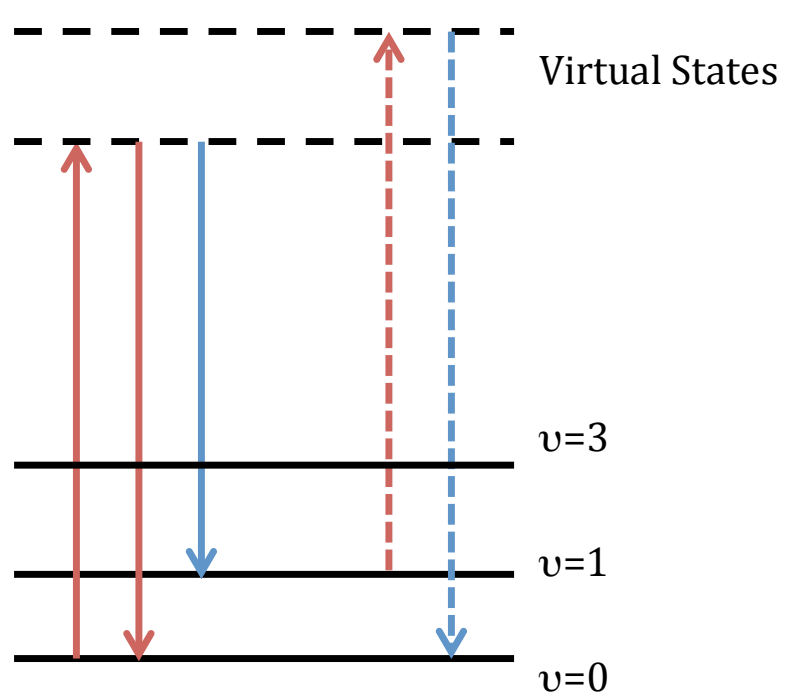

Figure 1: Incident radiation causes excitation into a virtual state with $\mathrm{E}=\mathrm{h} v_{\mathrm{ex}}$, indicated by the solid red line on the far left. When the molecule relaxes, if the photon emitted is equal to $\mathrm{E}=\mathrm{h} v_{\mathrm{ex}}$ then it is called Rayleigh scatter. The Rayleigh scattering is depicted as the second solid red line on the far left. The Stoke Scattering is the solid blue line that has the photon energy $=h\left(v_{\mathrm{ex}}-v_{\mathrm{v}}\right)$. The anti-Stokes Scattering needs the molecule to be in a higher vibrational state when excited and then the emitted photon can have the energy $=h\left(v_{\text {ex }}+v_{v}\right)$. 


\section{Surface Enhanced Raman Spectroscopy}

Surface enhanced Raman spectroscopy (SERS) was first documented in 1974

when Fleischamn et al. ${ }^{5}$ demonstrated that the Raman signal of pyridine adsorbed on an electrochemically roughened silver electrode that was many times greater than was expected. Because the voltage applied to the electrode affected the signal level, it was attributed to increased surface coverage of pyridine on the electrode. In the few years that followed, Van Duyne et al. managed to quantify the phenomenon and found that, rather than a concentration effect, the phenomenon was in fact an enhancement or increase in the Raman cross section of the adsorbed pyridine molecules of the shockingly large average magnitude of one million. ${ }^{6}$ This, in turn, led Van Duyne et al. ${ }^{6}$ to the conclusion that the increase in Raman cross section was due to the unique electromagnetic environment present in the roughened silver to which the pyridine was adsorbed. In the following year, Schatz et al. ${ }^{7}$ theorized that the enhanced Raman signal could be described by a frequency dependent coupled dipole mechanism wherein the electromagnetic resonance of conduction band electrons within the nanostructured silver electrode drove complementary dipolar oscillations in the adsorbed molecules. Numerous other observations ${ }^{8,9,10}$ were made in the subsequent years in order to elucidated the electromagnetic explanation for the Raman enhancement, which became universally known by the acronym SERS ${ }^{11}$ for surface enhanced infrared spectroscopy.

Over the years, groups have aimed to maximize the enhancement factor of SERS, 
with some reporting numbers as high as $10^{14} .{ }^{12}$ The race for the theory that would explain such high SERS enhancement factors was divided into two main groups of thought, an electromagnetic mechanism ${ }^{13,14,15}$ and a chemical mechanism ${ }^{16,17,18}$. The electromagnetic mechanism posits that the aforementioned electromagnetic resonance of the metal nanoparticle (now termed a localized surface plasmon resonance) underpins the enhancement and that it is independent of bonding between the analyte molecule and the metal surface. Using existing theoretical frameworks, this electromagnetic mechanism can be used to explain enhancement factors (EF) of between $10^{4}-10^{8}$, and depends approximately on the magnitude of the incident electric field $(E(\omega)$ raised to the fourth power (Equation 1). ${ }^{19}$

Equation 1. $\quad E F_{E M} \propto|E(\omega)|^{2}\left|E\left(\omega^{\prime}\right)\right|^{2} \approx|E(\omega)|^{4}$

In Equation $1 \mathrm{E}(\omega)$ is the frequency-dependent electric field at the incident frequency $\omega$, and $E\left(\omega^{\prime}\right)$ is the frequency-dependent electric field at the Stokes shift frequency $\omega^{\prime}$.

From this equation, one can understand why maximum $\mathrm{EF}_{\mathrm{EM}}$ is slightly blueshifted from the plasmon resonance peak (the peak in $\mathrm{E}[\omega]$ ). This is because the maximum $\mathrm{EF}_{\mathrm{EM}}$ should lie between the plasmon frequency and the Stokes frequency. The Stokes shift is normally small relative to the frequency of the laser; therefore equation 1 is usually approximated as $E F_{E M} \propto|E(\omega)|^{4} .{ }^{19}$ Hence SERS should be observable in those settings where localized (on nanoparticles or highly curved surfaces) or propagating (on flat surfaces) surface plasmons may be excited, a notion that is supported by experimental observations of intense SERS from many plasmonic systems 
including isolated and coupled nanoparticles and their assemblies.

There is still, however, some basis for the idea that the entirety of the SERS effect may also include a "chemical" contribution, (i.e., one that depends on the details of the bond formed between the adsorbate molecule and the metal). This idea includes the concepts of mixing of the electronic states of the molecule with those of the metal, and the attendant changes in symmetry and available electronic states through which the Raman scattering may couple. In support of this idea, for example, one may cite the dependence on electrochemical potential of the SERS enhancement ${ }^{20}$ and the presence of the many observations of SERS wherein the total enhancement greatly exceeds that which theoretically should be possible given a purely electromagnetic mechanism. Additionally, SERS has been observed in nonplasmonic metals, where the electromagnetic contributions should be much smaller. ${ }^{19}$

There also exists a resonance enhancement contribution to the Raman effect that may occur either alone or in combination with surface enhancement (i.e., SERS).

Resonance Raman differs from normal Raman in that the molecule is able to absorb the incident radiation via a normal electronic absorbance transition, and hence, rather than a virtual intermediate state, resonance Raman occurs via a conventional electronically excited state. In this mechanism, one often observes the relatively narrow-band Raman spectrum superimposed upon the fluorescent emission band of the molecule. ${ }^{4}$ However, since the fluorescent emission carries, at the minimum, a measure of shot-noise, which is proportional to the square root of the intensity of the fluorescent signal, this method is 
often of limited utility. However, when fluorescent emission may be suppressed, either by quenching via proximity to a metal surface, or in other time-resolved motifs, then electronic resonance may contribute substantially to the detectability of a Raman signal and may further multiply the large SERS cross sections. The resonance contribution to Raman enhancement is estimated to be of magnitude $10^{2}-10^{4} .^{2}$

An important theory of the underpinning of the chemical mechanism of SERS is the so-called charge-transfer mechanism. The charge-transfer mechanism occurs when the wave functions of the molecule and the metal overlap. In this case, the enhancement process occurs in a five-step manner. First, the metal absorbs the photon and excites the surface plasmon. The energy is then transferred to the molecule, which is used to excite the molecule into an excited electronic state. The molecule then relaxes and the energy, minus a vibrational quantum, is transferred back to the metal, which in turn re-radiates the new photon with the Stokes shifted frequency. Despite solid theoretical modeling, this mechanism remains controversial because of the difficulty in experimentally isolating charge transfer contributions. ${ }^{19}$

Many articles report a SERS enhancement factor, and therefore it is important to discuss the origin. It is difficult in most settings to establish the absolute value of a Raman scattering cross section. However, in those cases where a signal from the molecule under study can be collected both with and without an enhancing surface and at 
known concentrations, the relative enhancement factor may be computed as defined in

\section{Equation 2.}

Equation 2: $\quad E F=\frac{\left[\frac{I S E R S}{N_{a d s}}\right]}{\left[\frac{I_{N R S}}{N_{\text {vol }}}\right]}$

In this equation, the intensity of the SERS signal ( $\mathrm{I}_{\mathrm{SERS}}$ ) is divided by the number of molecules adsorbed to the nanoparticles $\left(\mathrm{N}_{\mathrm{ads}}\right)$ that are sampled. This number is then divided by normal Raman intensity ( $\mathrm{I}_{\mathrm{NRS}}$ ) for a given number of molecules in the excitation volume $\left(\mathrm{N}_{\mathrm{vol}}\right) .^{22}$

Nanoparticles are utilized in SERS because of a unique property called localized surface plasmon that arises from their small nature relative to the wavelength of light. Although SERS is not limited to nanoparticles, they are used because of their relatively easy synthesis and of the variety of geometries in which they appear, yielding a range of degrees of experimental freedom available to explore the optimization of SERS intensities.

\section{Localized Surface Plasmon Resonance}

Metal NPs have unique optical properties that are dramatically different from bulk metals of the same composition. ${ }^{23,24,25,26}$ This is evident, for example, when one looks at light coming from a ruby red piece of stained glass. The rich red color comes from $\mathrm{Au}$ 
nanoparticles dispersed throughout the glass matrix. ${ }^{27}$ While bulk Au has a yelloworange tint, a colloidal Au solution with nanoparticles sizes of ca. 20-40 nm typically is a distinctive wine or ruby red color. The color is attributable to a combination of intense absorption and scattering centered in the $500 \mathrm{~nm}$ wavelength range and which corresponds to the excitation of localized surface plasmon resonance (LSPR) that is unique to this size regime for gold. LSPR occurs when light of an appropriate wavelength excites a collective resonant oscillation of the conduction electrons within the metal particle. This effect imparts to the nanoparticles a large wavelength-dependent extinction (absorption plus scattering) equivalent to a molar extinction coefficient of $10^{11}$ $\mathrm{M}^{-1} \mathrm{~cm}^{-1}$ in certain cases. ${ }^{7}$ On a per-particle basis this means that a single $100 \mathrm{~nm} \mathrm{Ag} \mathrm{NP}$ scatters light with such efficiency that it appears as bright in an epifluorescence image as a collection of roughly one million ultra-fluorescent fluorescein molecules. ${ }^{29}$ Depending on their size, shape and composition, the extinction band of nanoparticles may cover a large range of the visual or near infrared optical spectrum. In many instances, synthetic adjustment of, for example, the length of a nanorod yields a plasmon extinction that is tunable across a significant range and may thus be selected so as to optimize its application to a particular laser excitation or other experimental need.

The unique optical properties of nanoparticles were first mathematically explained in 1908 by the German physicist Gustav Mie in what are called the Mie solutions in his honor. ${ }^{30}$ The Mie solutions are also referred to by some as the Mie equations, or Mie theory, and the attendant phenomenon is described as Mie scattering. For the remainder of this paper they will be referred to as the Mie solutions. The Mie 
solutions are a set of mathematical expressions that are solutions to Maxwell's electromagnetic equations in relation to the scattering of the electromagnetic radiation by a spherical particle with a radius, $r$, which is significantly smaller than the light wavelength, i.e. $r<<\lambda$. This condition insures that the electric field can be taken as a constant and also simplifies the solution as it does not present the possibility of an electrical multipole in the nanoparticle. This limiting case is referred to as the longwavelength approximation of the dipolar resonance. Equation 3 is the condensed form of the Mie solution for finding the extinction cross section for spherical nanoparticle dipole.

\section{Equation 3:}

$$
\sigma=\frac{9 \cdot V \cdot \varepsilon_{m}^{3 / 2}}{c} \cdot \frac{\omega \cdot \varepsilon^{\prime \prime}(\omega)}{\left(\varepsilon(\omega)^{\prime}+2 \cdot \varepsilon_{m}\right)^{2}+\varepsilon^{\prime \prime}(\omega)^{2}}
$$

$\sigma$ is the scattering cross section, $V=\frac{4}{3} \pi r^{3}$, is the particle volume, c is velocity of light, $\varepsilon_{\mathrm{m}}$ is the dielectric of the environment, $\omega$ is the angular frequency of the light, and $\varepsilon=\varepsilon(\omega)^{\prime}+\mathrm{i} \varepsilon(\omega)^{\prime \prime}$ is the complex dielectric of the metal. Succinctly stated, in this case the plasmon resonance will be centered on a light frequency where the real part of the complex dielectric of the metal is twice the magnitude of the dielectric constant of the medium, i.e. $\varepsilon(\omega)^{\prime}=-2 \varepsilon_{\mathrm{m}}$. From this equation, it is evident that the extinction cross section is dependent on the dielectric of the environment. Many groups have exploited this phenomenon by producing colorimetric sensors that are sensitive the surroundings of the particles. ${ }^{28,31,32,33}$ This equation also explains the cross section dependence of the volume of the particle and therefore explains the different colors associated with the different sizes of the particles. 
When referring to plasmon resonance in nanoparticles, it is more commonly called localized surface plasmon resonance (LSPR). LSPR is analogous to the surface plasmon excited by the evanescent wave irradiation in a planar metallic surface. The dissimilarity comes from the fact that the LSPR in a nanoparticle is not dependent on the grazing angle of the incoming electromagnetic wave in order to couple with the plasmon wave of the conduction electrons to cause resonance. Instead on a NP, the electric field of light causes the free electrons on the particle to become polarized. The fixed positive core of the particle creates a restoring force according to Coulomb's law. The net reaction is a dipolar oscillation of the free electrons, see Figure 2.

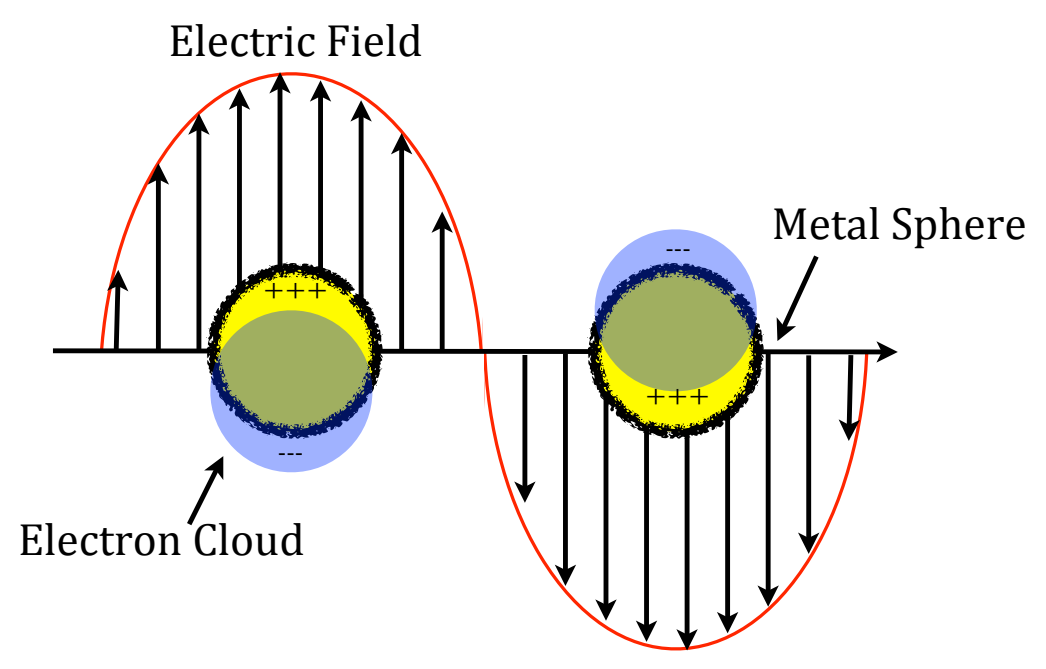

Figure 2: The electric field of the Electromagnetic radiation induces a polarization of the conduction electrons in the metal nanoparticles. The positive charge of the nanoparticle is stationary, while the negative charge of the electrons are stimulated by the electric field 
of the light. The positive charge on the particle applies a restoring force and thus causes an oscillation of the electrons.

\section{Colloid Interaction and Aggregation: DLVO Theory}

DLVO theory is an extension of the Debye-Huckel framework of the physical properties of ionic solutions. The important role of DLVO theory in nanoparticle research is its explanation of the stability and flocculation (aggregation) of colloidal particle solutions (suspensions) as a function of the ionic strength. The acronym is derived from the authors of the theory, Derjaquian, Landau, Verwery, and Overbeek honoring the co-discovery of the theory on by the former and latter pairs. According to DLVO, two forces govern the aggregation of neighboring particles, the Van der Waals $(\mathrm{vdW})$ attractive force, and the repulsive nature of the like-charged electrical double layer (EDL). Hence, DLVO provides a framework for understanding the crucial phenomenon of nanoparticle aggregation, a major determinant of SERS phenomena. The DLVO theory provides a net sum of attractive and repulsive forces as a function of NP-NP distance. The vdW attractive force term for two spherical nanoparticles is expressed in

\section{Equation 4.}

Equation 4: $\quad W(D)_{A}=\frac{-A}{6 D} \frac{R_{1}+R_{2}}{\left(R_{1} R_{2}\right)}$ 
In this equation, $R_{1}$ and $R_{2}$ refer to the radius of the respective nanoparticles. $A$ is the Hamaker constant, and D is the distance between the two nanoparticles. The repulsive term is given in Equation 5.

Equation 5: $\quad W(D)_{R}=\left(64 \pi k_{B} T R p_{\infty} \mathrm{Y}^{2} / \kappa^{2}\right) e^{-\kappa D}$

In Equation 5, $\mathrm{k}_{\mathrm{B}}$ is Boltzmann's constant, $\mathrm{T}$ is temperature (Kelvin), $\mathrm{R}$ is the radius of the radius of the nanoparticle, $p_{\infty}$ is the ionic strength of the solution, $\mathrm{Y}=\tanh \left(\frac{\mathrm{ze} \varphi_{0}}{4 \mathrm{k}_{\mathrm{B}}^{\mathrm{T}}}\right)$ is the reduced surface potential, $\varphi_{0}$ is the surface potential, $\kappa^{2}=$ $\sum_{i} \rho_{\infty_{i}} e^{2} z_{i}{ }^{2} / \epsilon \epsilon_{0} k_{B} T$ is the Debye sceening length, and $\mathrm{D}$ is the distance between the particles. To solve for the interaction potential of the two nanoparticles as a function of distance, $\mathrm{vdW}$ and EDL are summed:

Equation 6: $\quad W(D)=W(D)_{A}+W(D)_{R}$

DLVO states that when interaction potenital is negative, $W(D)_{A}>W(D)_{R}$, the neigbhoring nanoparticle will have a higher probablity of flocculating, and illustrates the role of ionic strength in promting this. Thus a critical concentration may be defined at which $\mathrm{W}(\mathrm{D})=0$.

\section{Experimental}

\section{Particle Synthesis}

Gold core silver shell nanoparticles were prepared using a chemical metal reduction procedure following Liu and Han. ${ }^{34}$ Gold nanoparticles were synthesized first 
using the single-phase water based Turkevich method. ${ }^{35}$ Our exact protocol begins with the addition of $8.8 \mathrm{mg}$ of potassium tetrachloroaurate to $47.5 \mathrm{~mL}$ of 4.5 micron-filtered water in a scrupulously cleaned $100 \mathrm{~mL}$ beaker and large Teflon coated stir bar. The temperature was allowed to rise gradually to boiling during the preparation of the a sodium citrate dihydrate solution, which was then prepared by dissolving $7.4 \mathrm{mg}$ in 2.5 $\mathrm{mL}$ of water, which was then placed alongside the tetrachloroaurate solution on the hot plate to warm slightly below boiling. Once the potassium tetrachloroaurate solution was boiling, the heated sodium citrate dihydrate was added under vigorous stirring by the Teflon-coated magnetic stir bar. The solution was allowed to boil and stir for 60 minutes during which time the color changed from a pale yellow, to a dark gray, and finally to a ruby red, after which time the heating element was turned off and the solution was allowed to cool gradually to room temperature over 30 minutes, maintaining the vigorous stirring during the cooling process. During this time the citrate reduces $\mathrm{Au}^{3+}$ to $\mathrm{Au}^{0}$ and causes nucleation of the Au metal to which more Au can crystallize onto and promote growth of the nanoparticle. Excess citrate, in turn, then serves to partially passivate (i.e., cap) the incipient particle surface thus moderating and controlling particle growth. The particles also obtain a negative charge during growth and thus do not tend to aggregate. The UV-visible absorption spectra of Au NP solutions were then acquired (Cary-50 Bio, $200-750 \mathrm{~nm})$ and the absorption band shapes and peak wavelengths $\left(\lambda_{\operatorname{MAX}} \sim 518 \mathrm{~nm}\right)$ checked to confirm their conformity to expected values. Au NP solutions were then stored at $4^{\circ} \mathrm{C}$ overnight before subsequent processing. 
Synthesis of the silver shell encasing the Au nanoparticles was implemented within 24 hours of the Au core synthesis. In this process, $20 \mathrm{~mL}$ of the $\mathrm{Au}$ citrate nanoparticle solution were mixed with $3 \mathrm{ml}$ of $0.1 \mathrm{M}$ ascorbic acid in an Erlenmeyer flask. In a separate beaker, $25 \mathrm{~mL}$ of $1 \mathrm{mM} \mathrm{AgNO} 3$ was made. The $\mathrm{AgNO}_{3}$ is then added to the stirring Au citrate solution at a rate of one drop per 30 seconds. This was accomplished with a peristaltic pump to insure a consistent rate of addition. In this procedure, the ascorbic acid reduces the $\mathrm{Ag}^{+}$into $\mathrm{Ag}^{0}$ that will crystallize on the Au nanoparticles in the solution. The degree of coating can be varied based on the amount of $\mathrm{AgNO}_{3}$ added to the solution. Since the Ag is coating the Au nanoparticles that are already in solution, the concentration of nanoparticles in solution is conserved from the Au nanoparticle synthesis. ${ }^{34}$

\section{Stock Solutions}

Stock solutions of Rhodamine 6G (R6G, Acros Organics) and 4-mercaptopyridine (MPY, Sigma-Aldrich) at 1, $0.1 \ldots 0.001 \mathrm{mM}$ were prepared by serial dilution of a 10 $\mathrm{mM}$ stock solution (22.18 $\mathrm{mg}$ R6G or $5.58 \mathrm{mg}$ MPY in $5 \mathrm{~mL}$ water) yielding solutions labeled A,B ... E respectively. All solutions are diluted with water purified by reverse osmosis, deionization and purification (Millipore Proguard 2) and 4.2 micron membrane filtration.

\section{Instrumental Apparatus}


Raman spectra were collected using an Enwave Optronics EZ-Raman spectrometer employing a $785 \mathrm{~nm}$ frequency stabilized, narrow linewidth 0-300 $\mathrm{mW}$ fiber coupled diode laser. In this instrument detection is via a high sensitivity TE-cooled CCD spectrograph with a useful spectral range of 100 to $3300 \mathrm{~cm}^{-1}$ (Stokes only) and an average optical resolution of $\sim 7 \mathrm{~cm}^{-1}$.

UV-vis absorbance spectra were obtained concurrently with Raman spectra using an absorbance spectrometer of local construction which employs an Ocean Optics LS1 tungsten halogen light source, coupled via $400 \mu$ m multimode fiber optic cable to a focusing lens. Visible light is focused into the center of the cuvette and collected with a matched lens and fiber optic cable opposite the cuvette and is then collected by an Ocean Optics USB 650 Red Tide spectrometer. Locally written software (LabView 8.2) is used to compute, display, and store absorbance spectra.

\section{Procedure}

To ensure that there is zero cross contamination between experiments, the quartz cuvette and magnetic stir bar are both cleaned with aqua regia and rinsed copiously with water before each experiment. The tungsten halogen lamp is allowed to warm up for 5 minutes before initial scans. Once this time has expired, water is placed in the cuvette to record a reference for the absorbance measurements. 
Nanoparticles were diluted with a 1:1 ratio of nanoparticles to water for each of the experiments as the undiluted solutions present too great an absorbance. During measurements, solutions within the cuvette were actively and vigorously stirred $(6 \mathrm{~mm} \mathrm{x}$ $3 \mathrm{~mm}$ Teflon coated magnetic stir bar) in order to ensure solution homogeneity. Addition of R6G and other titrants was done using a dedicated set of autopipettors (Fisher Pipetman).

Most titrations follow Table 1, and span 5 orders of magnitude in titrant concentration, beginning with $0.001 \mu \mathrm{M}$ and ending at $250 \mu \mathrm{M}$. Table 1 shows the precise amount of each addition of the analyte and the resulting total titrant concentration. After each addition of titrant, a one-minute adsorption time was allowed followed first by the acquisition of the Raman (4 spectra serially averaged from 4 second integrations and at $1 \mathrm{~cm}^{-1}$ nominal resolution) and then of the UV-Vis spectra (350-1000 nm at $1 \mathrm{~nm}$ nominal resolution), were acquired within a time interval of roughly 1 minute. 
Table 1: Universally applied spike titration series used for all analytes. Specified volumes were pipetted into a $2.00 \mathrm{~mL}$ of nanoparticle solution in stirred cuvette during the course of the acquisition of the series of Raman and extinction spectra. The titration is designed to cover a very large range in analyte concentration ( 5 orders of magnitude) from starting with $0.001 \mu \mathrm{M}$ and ending at $250 \mu \mathrm{M}$.

\begin{tabular}{|c|c|c|c|c|}
\hline serial \# & $\begin{array}{c}\text { spike vol } \\
\text { uL }\end{array}$ & $\begin{array}{l}\text { cum tot } \\
\text { volume }\end{array}$ & Vial & \begin{tabular}{|c} 
corrected \\
final conc \\
$\mu \mathrm{M}$ or $\mu \mathrm{g} /$ \\
$\mathrm{mL}$
\end{tabular} \\
\hline 0 & 2.0 & 2.002 & $E$ & 0.00100 \\
\hline 1 & 3.2 & 2.005 & $E$ & 0.00258 \\
\hline 2 & 5.0 & 2.010 & E & 0.00507 \\
\hline 3 & 8.0 & 2.018 & $E$ & 0.00900 \\
\hline 4 & 12.62 & 2.031 & $E$ & 0.0152 \\
\hline 5 & 2.0 & 2.033 & D & 0.0250 \\
\hline 6 & 3.2 & 2.036 & D & 0.0405 \\
\hline 7 & 5.0 & 2.041 & D & 0.0650 \\
\hline 8 & 8.0 & 2.049 & D & 0.104 \\
\hline 9 & 12.6 & 2.062 & D & 0.164 \\
\hline 10 & 2.0 & 2.064 & C & 0.261 \\
\hline 11 & 3.2 & 2.067 & C & 0.414 \\
\hline 12 & 5.0 & 2.072 & c & 0.655 \\
\hline 13 & 8.0 & 2.080 & c & 1.04 \\
\hline 14 & 12.6 & 2.092 & C & 1.63 \\
\hline 15 & 2.0 & 2.094 & B & 2.59 \\
\hline 16 & 3.2 & 2.097 & B & 4.09 \\
\hline 17 & 5.0 & 2.103 & B & 6.47 \\
\hline 18 & 8.0 & 2.110 & B & 10.2 \\
\hline 19 & 12.6 & 2.123 & B & 16.1 \\
\hline 20 & 2.0 & 2.125 & A & 25.5 \\
\hline 21 & 3.2 & 2.128 & A & 40.4 \\
\hline 22 & 5.0 & 2.133 & A & 63.8 \\
\hline 23 & 8.0 & 2.141 & A & 101 \\
\hline 24 & 12.6 & 2.154 & A & 159 \\
\hline 25 & 20.0 & 2.174 & A & 249 \\
\hline
\end{tabular}

\section{Results and Discussion}

\section{Nanoparticle synthesis}

Each batch of newly synthesized nanoparticles was analyzed by UV-vis absorbance. Figure 3 illustrates a typical Au nanoparticle extinction (absorbance plus scattering loss) spectrum. The absorbance peak in the $530-540 \mathrm{~nm}$ range is characteristic of the dipolar plasmon resonance of the spherical particles, and the peak magnitude and 
wavelengths are used as a control check for the successful synthesis of $\mathrm{Au}$ nanoparticles. ${ }^{36}$ The Mie solutions provide an excellent prediction of the NP extinction spectra. ${ }^{37}$ In particular, the dielectric function of the Ag shell is predicted and observed to impose a thickness-dependent blue shift in the dipolar plasmon band. ${ }^{38}$ The peak wavelength for example is indicative the thickness of the Ag shell and makes an excellent diagnostic of the NP synthesis. 


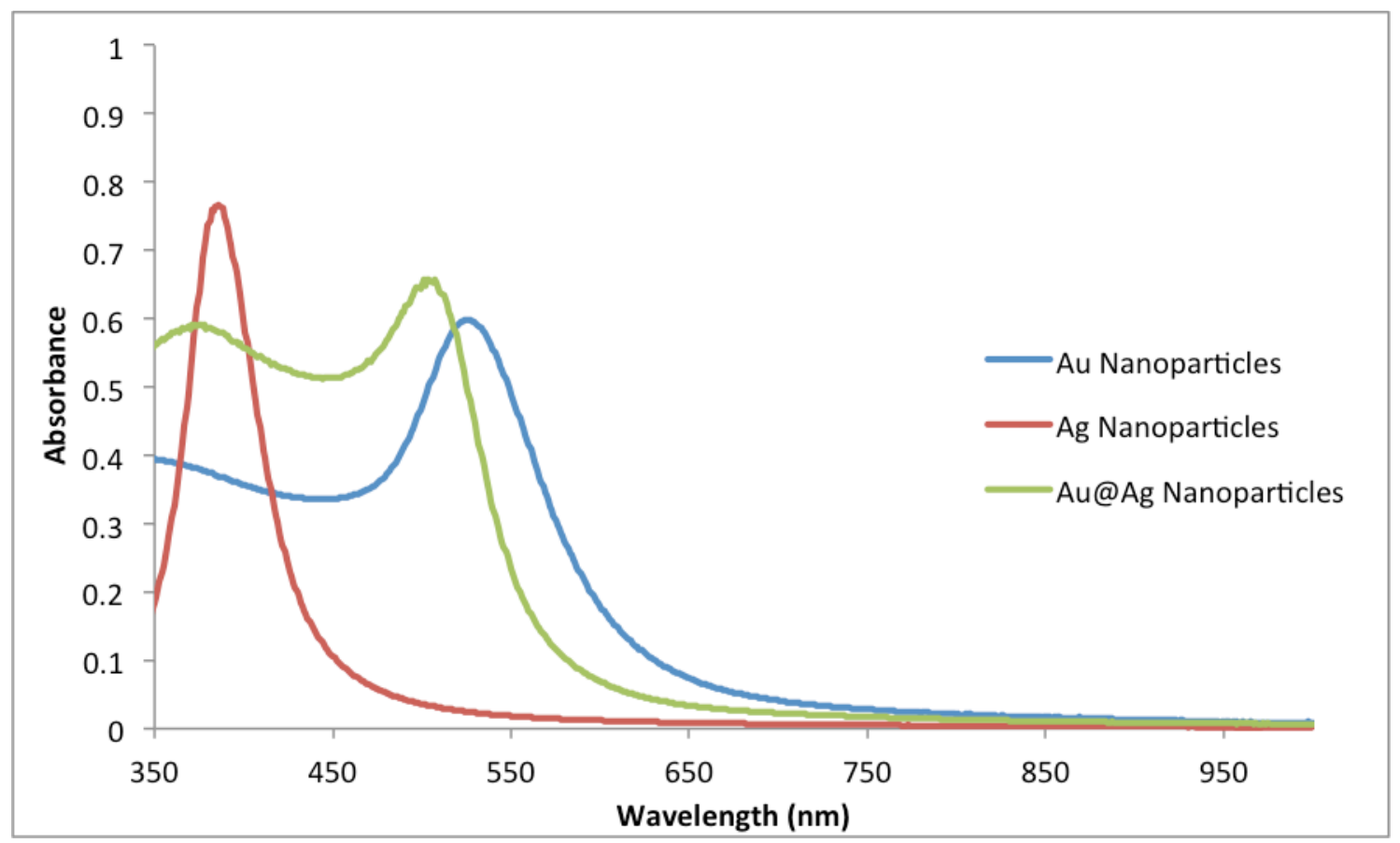

Figure 3: The blue plot is Au nanoparticles with the maximum absorbance at $530 \mathrm{~nm}$. The red line is a solution of Ag nanoparticles with a maximum absorbance of $388 \mathrm{~nm}$. The green line is the absorbance spectra of $\mathrm{Au} @ \mathrm{Ag}$ nanoparticles, which present two peaks at 508 and $380 \mathrm{~nm}$.

\section{Testing SERS Activity}

Freshly prepared nanoparticle specimens were assayed for SERS activity by a single addition of the SERS label such as R6G at a high concentration. For SERS active particle solutions, titration experiments were carried out. Figure 4 illustrates that at $2 \mu \mathrm{M}$, the nanoparticles are needed to observe the Raman scattering of R6G. 


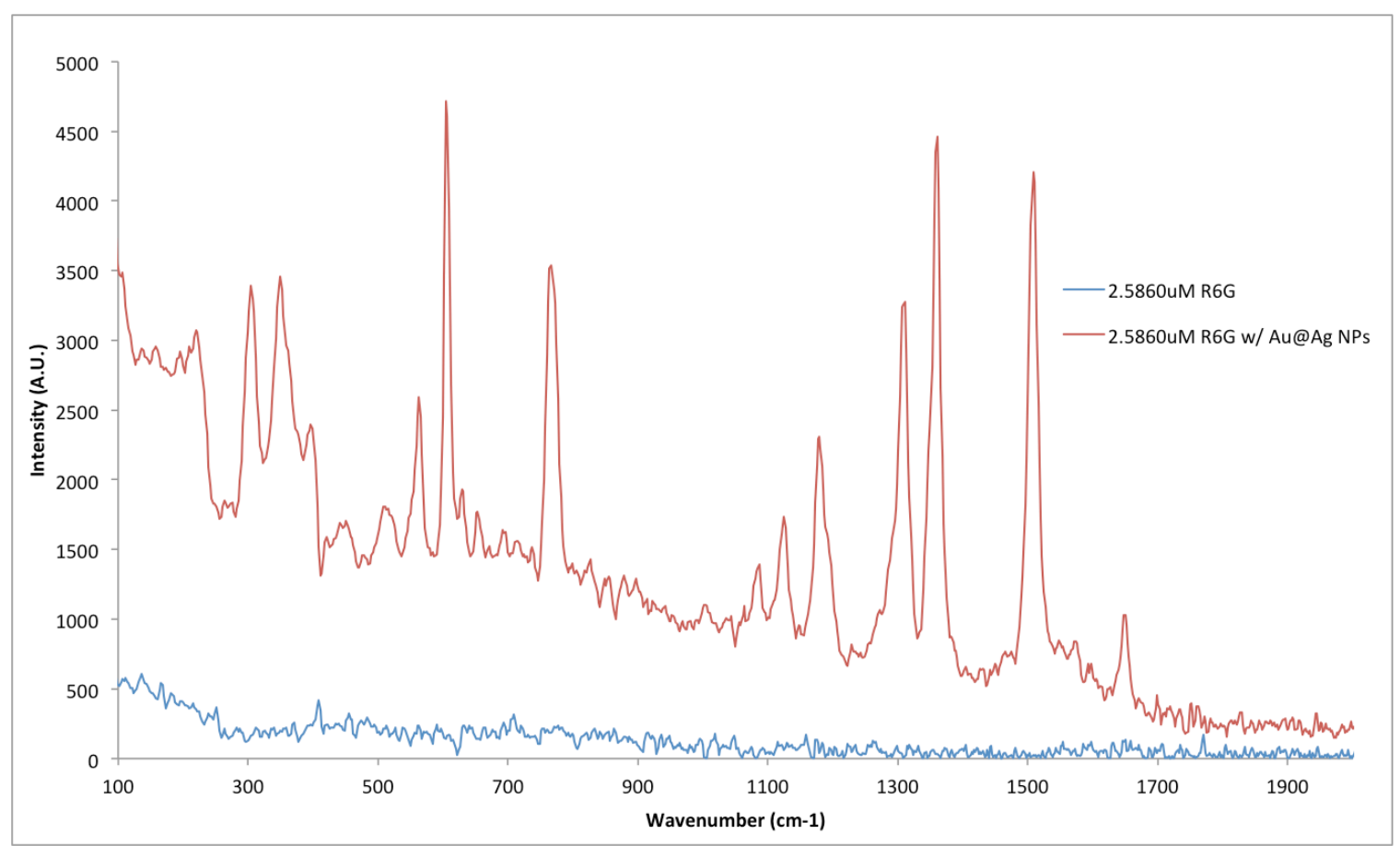

Figure 4: Raman spectra of $2.6 \mu \mathrm{M}$ R6G; Blue line is $2.6 \mu \mathrm{M}$ R6G in water, red $2.6 \mu \mathrm{M}$ R6G in $1 \mathrm{~mL}$ of water and with $1 \mathrm{~mL}$ of as-prepared Au@Ag nanoparticles.

\section{Comparing AuVsAu@Ag}

It was important to choose nanoparticles that present the greatest enhancement of SERS signal. Figure 5 shows a typical comparative test between $\mathrm{Au}$ and $\mathrm{Au} @ \mathrm{Ag}$ nanoparticles with $1 \mu \mathrm{M}$ R6G. In the great majority or our experiments, Au@Ag nanoparticles are similarly superior SERS enhancers relative to pure Au or Ag particles as is documented by a range of groups. ${ }^{34,38}$ Due to the strong SERS properties of $\mathrm{Au} @ \mathrm{Ag}$ nanoparticles, nanoparticles of this composition are used throughout the rest of the experiments. 


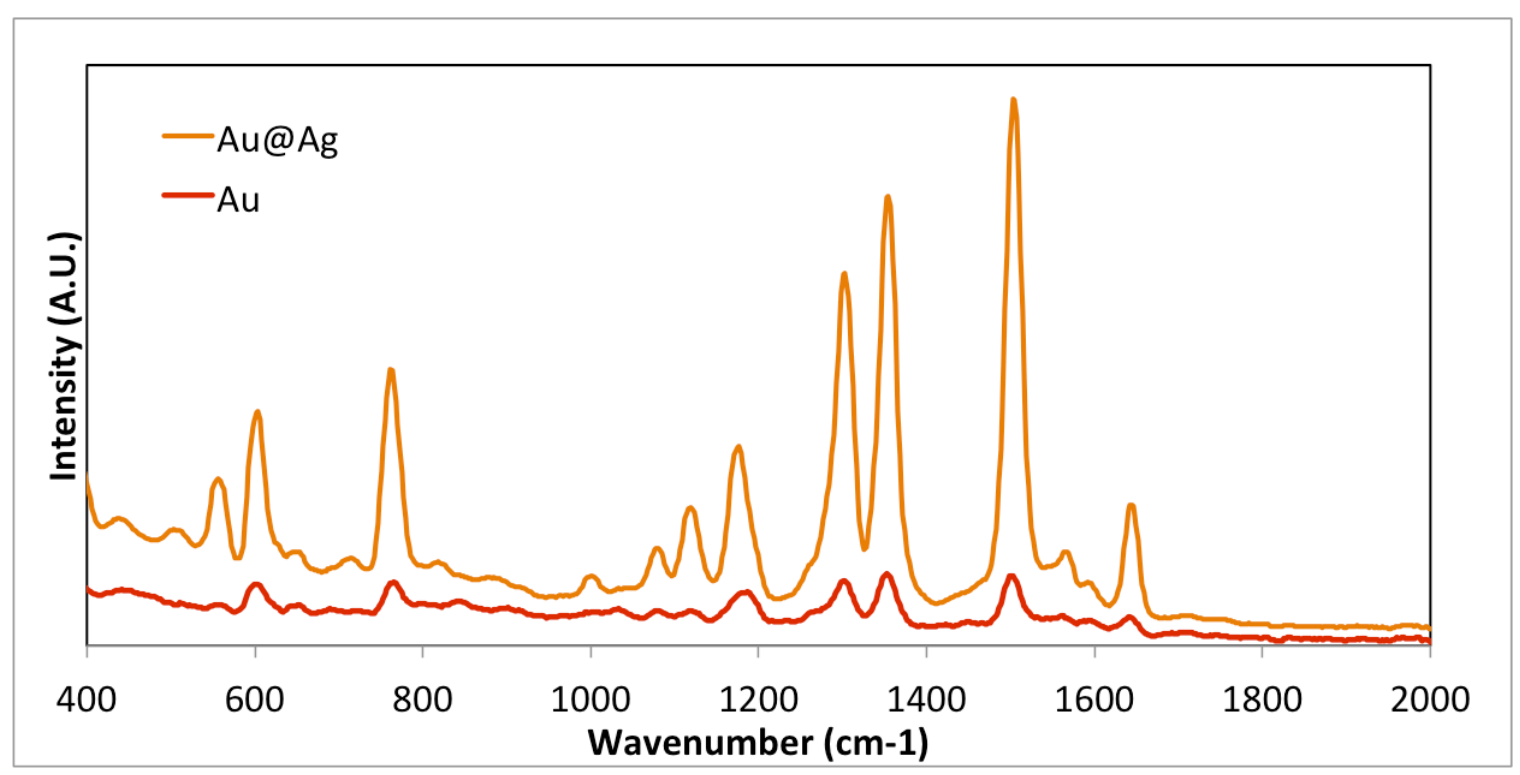

Figure 5: SERS of R6G of the same concentration differing only in the composition of the nanoparticle. The orange plot is with an $\mathrm{Au} @ \mathrm{Ag}$ nanoparticle solution. The red plot is a pure Au nanoparticle solution.

\section{Nanoparticle Titration with R6G}

In numerous replicate experiments, $\mathrm{R} 6 \mathrm{G}$ was titrated into a $2 \mathrm{~mL}$ solution of Au@Ag nanoparticles as illustrated in Table 1. This was done to have a consistent concentration scale and to span a wide range of concentrations. Figure 6 superposes Raman spectra recorded at each step in the titration, with lower concentrations in red and spanning the spectrum to the highest concentrations in blue. 


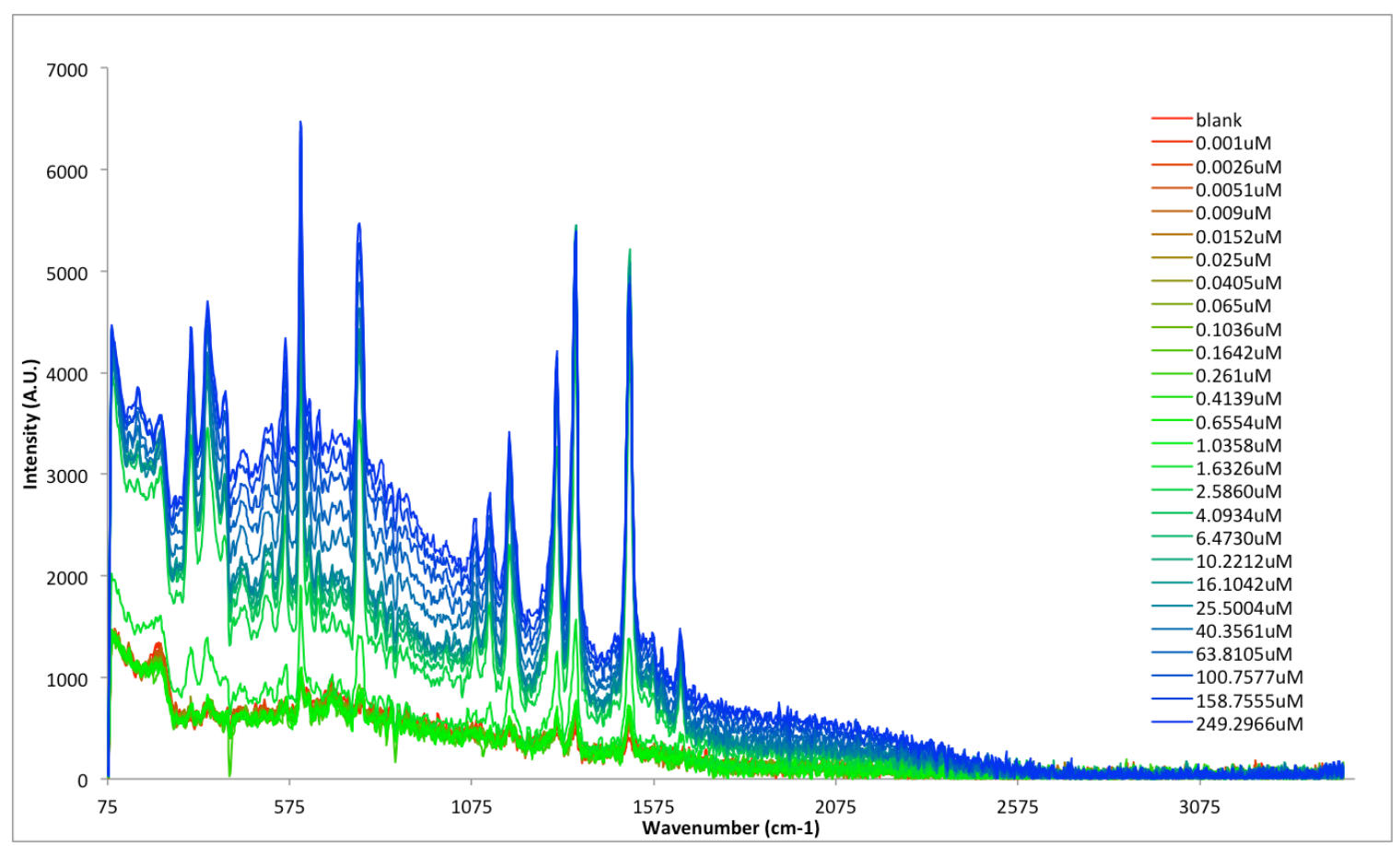

Figure 6: Raman spectra recorded during titration of $\mathrm{Au} @ \mathrm{Ag}$ with $\mathrm{R} 6 \mathrm{G}$. One mL of water and 1mL of $\mathrm{Au} @ \mathrm{Ag}$ nanoparticles were titrated with a series of increasingly concentrated R6G solutions. The graph is color-coded with the blank, $1 \mathrm{~mL}$ water and 1 $\mathrm{mL}$ of nanoparticles, being red and increasing concentrations progressing into the blue as indicated in the legend. 


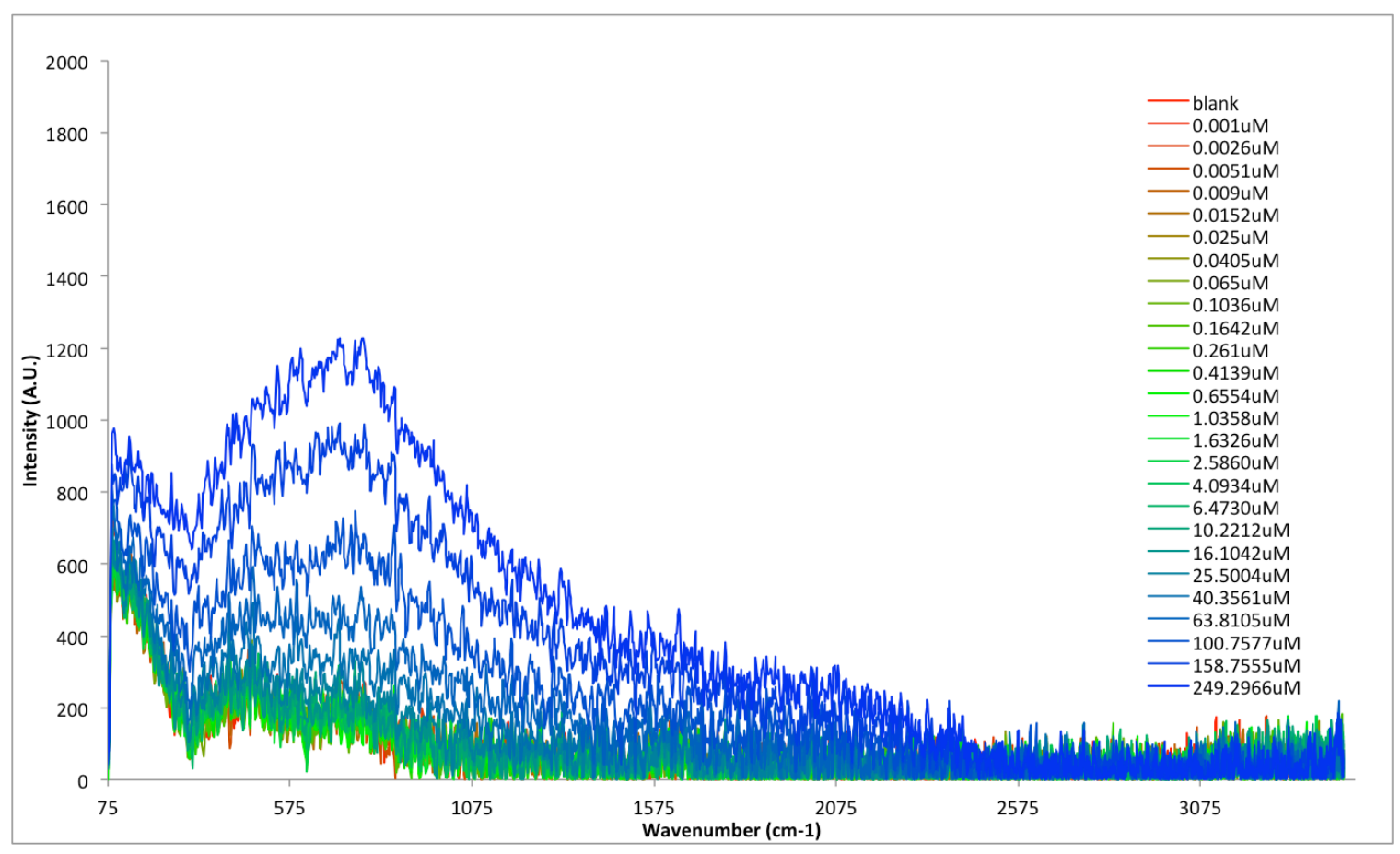

Figure 7: Raman spectra acquired as R6G is titrated into pure water illustrating.

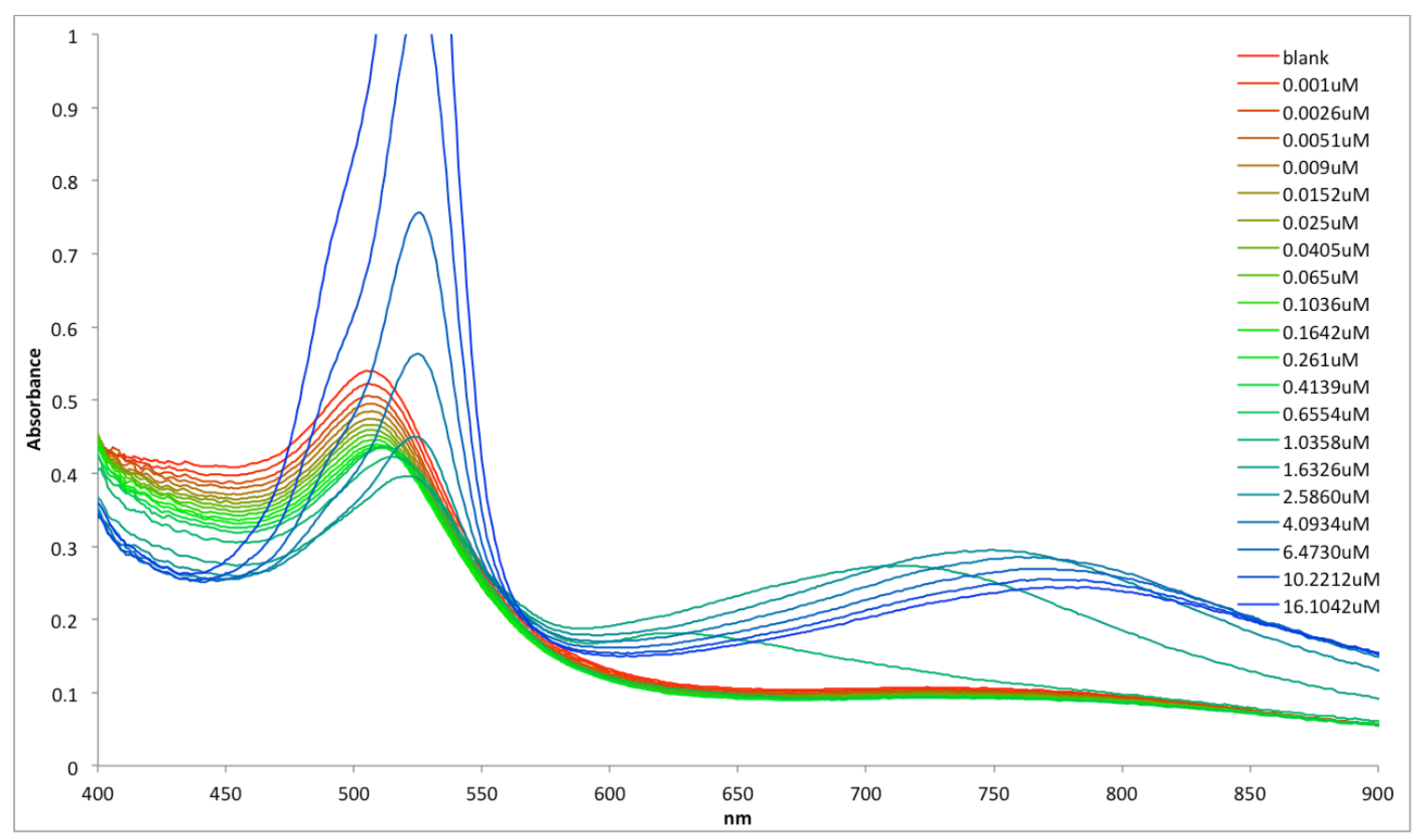

Figure 8: Vis-NIR spectra acquired as Au@Ag is titrated with R6G. 


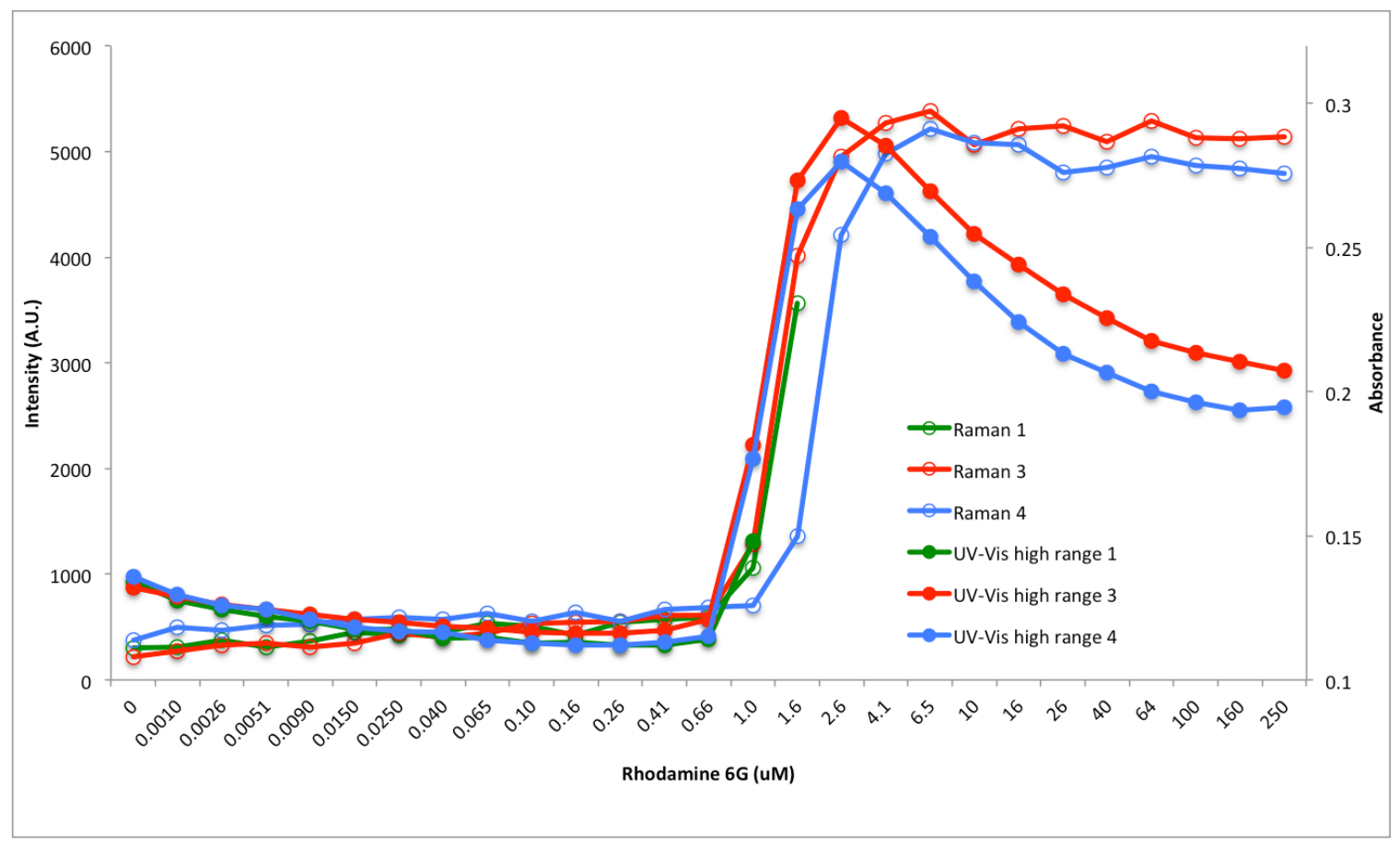

Figure 9: Replicate titrations of $\mathrm{Au} @ \mathrm{Ag}$ in pure water with R6G. Peak intensity of 1508 $\mathrm{cm}^{-1}$ band (left axis, closed symbols) is plotted alongside $\mathrm{A}_{\mathrm{MAX}}$, the maximum absorbance value found within the spectral range of $600-850 \mathrm{~nm}$, and belonging to the plasmon resonance of dimeric or aggregated nanoparticles.

For the R6G system, the onset of SERS appears abruptly following the $1 \mu \mathrm{M}$ point in the titration and peaks in amplitude by roughly $4 \mu \mathrm{M}$. This type of behavior is clearly indicative of some relatively abrupt physical transformation, such as a phase change, precipitation, or other critical phenomenon, and is quite distinct from an adsorption isotherm which, (e.g., in the case of a Langmuir process) would span roughly 2 orders of magnitude in adsorbate (R6G) concentration before fully saturating a surface. Careful inspection of Figure 6 shows that between 1.0 and $1.7 \mu \mathrm{M}$, the intensity of the 
SERS jumps dramatically. Figure 7 illustrates the Raman fluorescence background signature of $\mathrm{R} 6 \mathrm{G}$ in water.

Changes in the UV-visible spectra of the nanoparticle solutions appear with near perfect correlation to the SERS onset. The abrupt appearance of the near infrared peak, likely the longitudinal plasmon resonance, along with the decline in intensity of the $\sim 450 \mathrm{~nm}$ dipolar plasmon for free particles, produces a visible orange to gray/blue color transition in the solution. Figure 8 shows the UV-vis data acquired during the titration illustrated in Figure 6. Three important pieces of information can be taken from these figures. First, there is a gradual red shift of the $508 \mathrm{~nm}$ peak as the R6G is titrated into the solution. This is indication that there is an increase in the dielectric constant around the nanoparticles that is caused by the coating of the analyte. Increasing the near-particle dielectric will cause a red-shift in the plasmon resonance and thus a shift in the absorption and scattering that is seen in the $\Delta \lambda_{\mathrm{MAX}}$ in the UV-Vis spectrum. This effect is predicted by the dipolar term of the Mie solutions for the total extinction ( $\sigma$, scattering plus absorption) of the nanoparticle. ${ }^{30}$

Further into the titration, a second peak emerges in the $700-900 \mathrm{~nm}$ range. This peak is ascribed to the formation of particle dimers or higher aggregates. For a pair of particles between which the interparticle distance has decreased beyond a critical point, the plasmons from the different nanoparticles interact in such a way that electromagnetically the two particles behave as though they were a single larger particle with distinct longitudinal and transverse axes. The resulting, distinctive spectrum is 
illustrated in Figure 10. Therefore with the appearance of a peak within the 700-900 nm range, the conclusion can be made that there is formation of aggregates within the solution. ${ }^{37,39-42}$

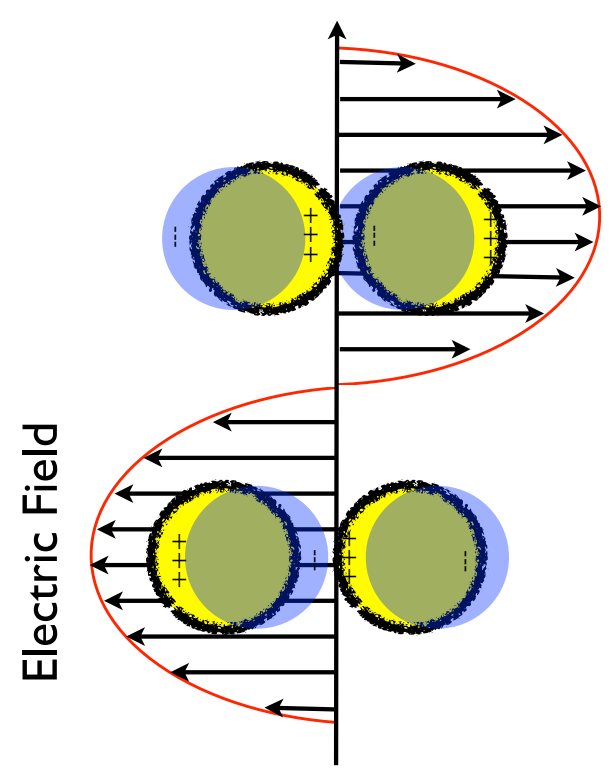

Figure 10: Illustration of the formation of interparticle "hot spots" when the light electrical field is parallel to interparticle axis. In this configuration, the plasmon oscillations of adjacent particles interact. The electric field peaks in the interparticle gap, creating, in is believed, SERS "hot spots" where Raman enhancement can reach enormous $\left(10^{6}-10^{9}\right)$ values.

Referring to Figure 8 for the moment, one may observe that beyond approximately $2 \mathrm{mM}$, a peak with $\lambda_{\mathrm{MAX}}=530 \mathrm{~nm}$ appears prominently, which is purely due to the absorption of free R6G. In Figure 11 we make an approximate correction for this interference by subtracting the corresponding blank (zero nanoparticle titration) R6G 
absorption spectrum from the nanoparticle one, to better reveal the plasmon band in the $505 \mathrm{~nm}$ range. This is admittedly an overcorrection as it assumes that $\mathrm{R} 6 \mathrm{G}$ adsorption has no influence on the R6G absorbance. Figure 12 Illustrates the case of titration with 4-mercaptopyridine (MPY). MPY offers a similar example of how aggregation may influence the Au@Ag plasmonic spectrum, and in this case, it is free of spectral interference in the region of the dipolar resonance near $505 \mathrm{~nm}$ as MPY does not significantly absorb or scatter light a $\lambda>450 \mathrm{~nm}$. The MPY titration in Figure 12 illustrates the prominent appearance of what appears to be a longitudinal dipolar peak centered near $\lambda=785 \mathrm{~nm}$, which arises accompanied by the diminution of the modestly red-shifted transverse dipolar band near $525 \mathrm{~nm}$. The conservation of the $525 \mathrm{~nm}$ band is consistent with observations of nanoparticle aggregates and nanorod spectra and illustrates the geometrical sensitivity of the plasmonic spectra of nanoparticles and their assemblies. The transverse dipolar plasmon mode is depicted in Figure 13. 


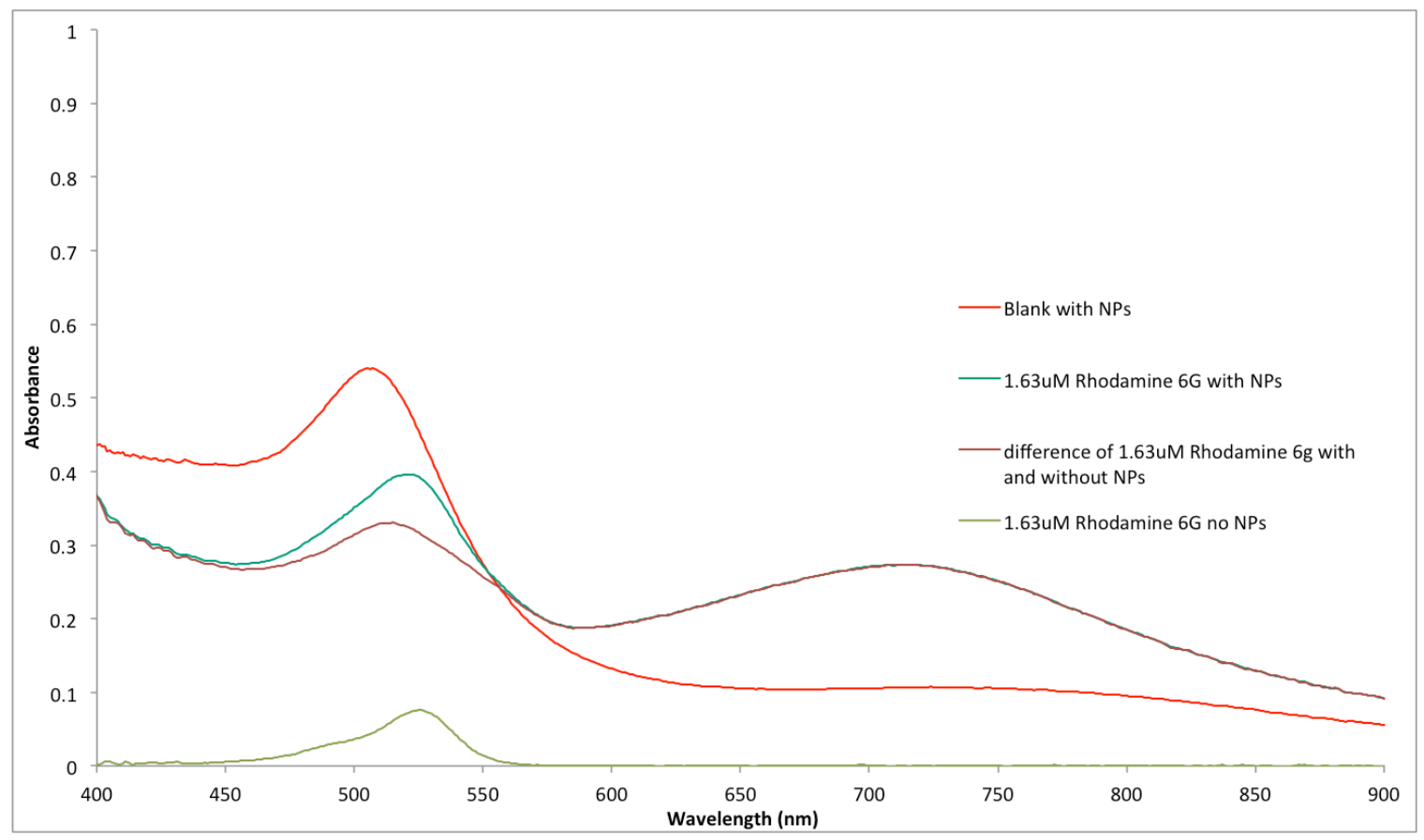

Figure 11: Nanoparticle aggregation spectra in the UV-Vis. This spectrum shows that when $\mathrm{R} 6 \mathrm{G}$ reaches a concentration of $1.63 \mu \mathrm{M}$, a second broad peak in the $750 \mathrm{~nm}$ range appears. The formation of a peak in this range indicates nanoparticle aggregation within the solution. The green line is $1.63 \mathrm{mM} \mathrm{R6G}$ without nanoparticles. The difference between the $1.63 \mathrm{mM}$ R6G with nanoparticle and the $1.63 \mathrm{mM}$ R6G solution is the brown line. 


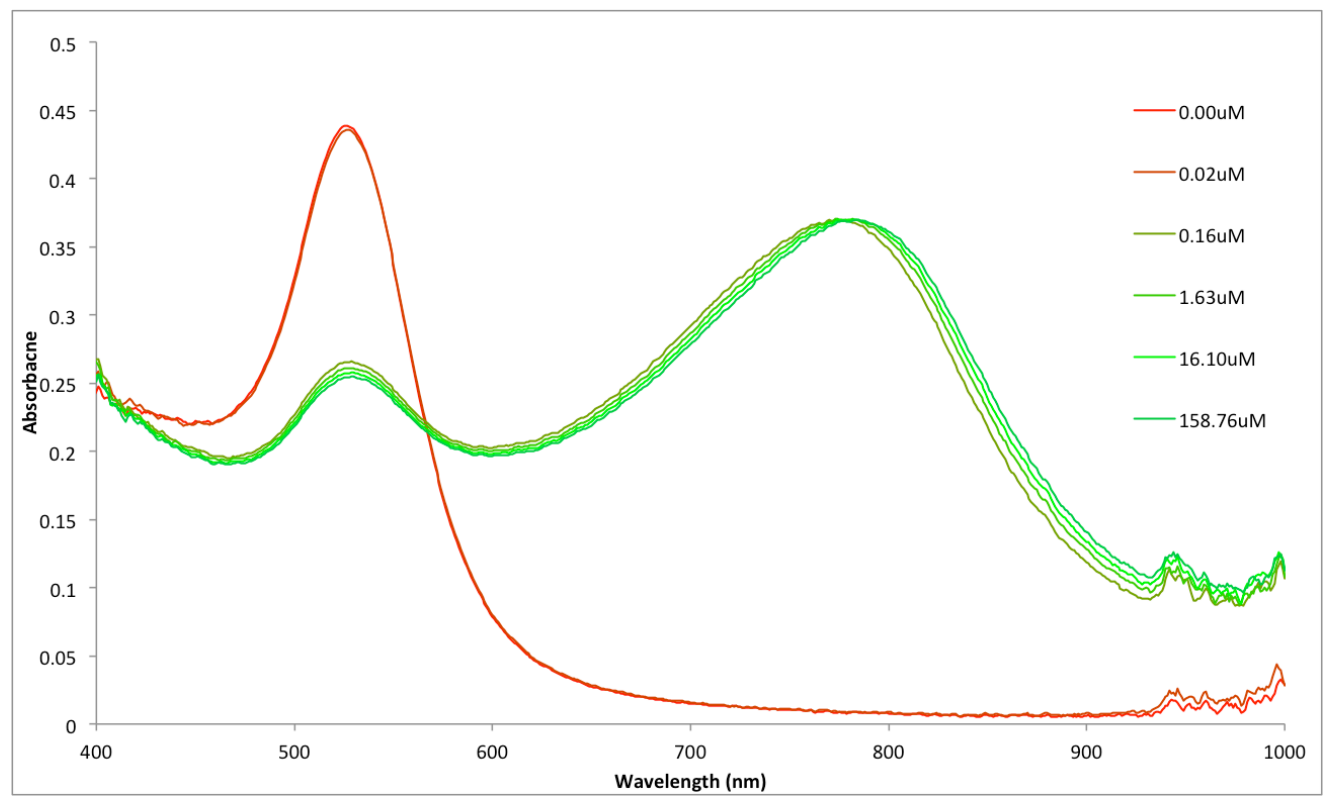

Figure 12: 4-Mercaptopyridine titrated into an Au nanoparticle solution. As the titration of 4-Mercaptopyridine reaches $1.63 \mu \mathrm{M}$, the appearance of a second peak at the $800 \mathrm{~nm}$ range indicates that the nanoparticles aggregated.

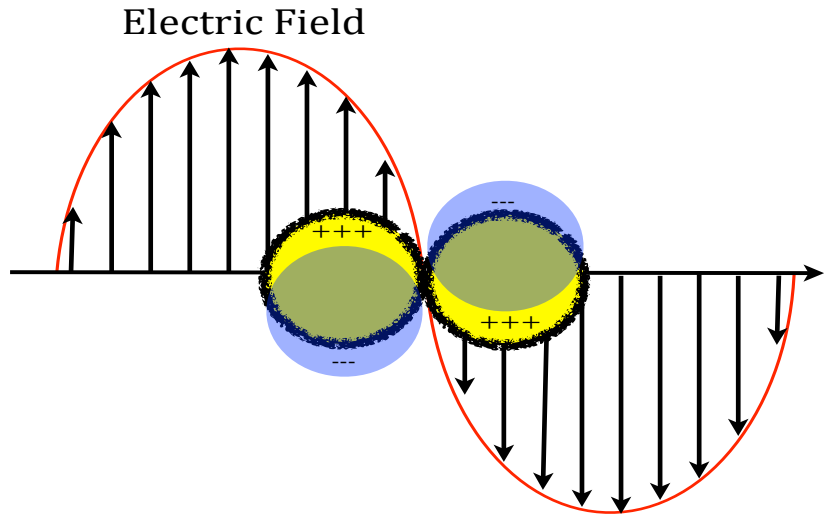

Figure 13: Illustration of the weakly interacting transverse dipolar mode that is created in the nanoparticle dimers when light electric field is orthogonal to the interparticle axis. 
Combining the information that is gained from both of these methods, one can make the argument that in the case of $\mathrm{R} 6 \mathrm{G}$, the aggregation of the nanoparticles and the onset of SERS are closely correlated. As illustrated in Figure 9, the onset of SERS and the appearance of the longitudinal plasmon peak at 700-900 $\mathrm{nm}$ range appear nearly simultaneously. In this case, it would appear likely that the intense SERS is a consequence of the formation of interparticle "hot spots" - interparticle interstices where optical electromagnetic fields are highly amplified and which lead to high SERS intensities. ${ }^{43-46}$ Such a possible "hot spot" is depicted in Figure 10, which shows that the interparticle gap may host a small region wherein the positive and negative poles of neighboring particles are separated by a very small dielectric gap, which will have a correspondingly large electric field. Existing literature includes numerous computational studies on idealized rod and sphere-pair geometries indicating good correlation between experimental and theoretical extinction spectra. ${ }^{47,48}$

Figure 9 also clearly illustrates that the SERS signal intensity begins to gradually diminish only very shortly after its peak. This diminution may originate from either a. source absorption, b. a decline in SERS emission or c. absorption of SERS emission. Since absorbance profiles (closed symbols) indicate a relatively flat profile in the NIR $(600-850 \mathrm{~nm})$ region $\left(1_{\text {SOURCE }}=785 \mathrm{~nm}, 1_{\text {SERS }}=890 \mathrm{~nm}\right)$ it seems likely that this diminution is due to changes in SERS emission. Marinica et al. ${ }^{49}$ theorize that the decrease in the signal is essentially due to the closing of interstitial gaps below an optimal size. The rationale is that below a certain sub-nanometer gap size, electrons begin to 
tunnel between particles and the polarization is reduced, and is supported by calculations but not direct experimental evidence of the tunneling. Another possible reason for the degradation of the SERS signal after the onset may be the formation of higher aggregates (trimers, tetramers etc.). Wustholz et al. ${ }^{29}$ showed that higher order aggregates produce little signal enhancement beyond the initial dimer formation. Therefore, it is logical to conclude that in the case of a solution undergoing extended aggregation, the average observed SERS intensity may fall as a result of source and emission absorption as hotspots are occluded within larger structures.

\section{Sodium Chloride Induced Aggregation}

Since aggregation appears to drive the activation of SERS in the R6G-Au@Ag system, a set of experiments wherein aggregation was triggered by the nearly SERS silent electrolyte sodium chloride. This alternative method of inducing aggregration was implemented at various points before and after the addition of R6G to nanoparticle solutions in order to examine the possibility of observing R6G SERS spectra at concentrations below those at which R6G itself appears to trigger the concomitant aggregation and SERS described above.

DLVO theory predicts that, for the parameters of $20 \mathrm{~nm}$ radius citrate capped $\mathrm{Ag}$ nanoparticles at $25^{\circ} \mathrm{C}$, the critical coagulation centration is $c a .120 \mathrm{mM}[\mathrm{NaCl}]$. In experiments used below intended to trigger either specific effects due to $\mathrm{Na}^{+}, \mathrm{Cl}^{-}$or aggregation, 10,100 or $150 \mathrm{mM}[\mathrm{NaCl}]$ is employed. 
In the first experiment, (red trace) a single spike was used to raise [R6G] to 0.125 $\mu \mathrm{M}$ R6G, then a second spike was used to raise the solution ionic strength to $150 \mathrm{mM}$ $[\mathrm{NaCl}]$, and the Raman and UV-VIS spectra were collected. For the system containing only $0.125 \mu \mathrm{M}$ R6G and Au@Ag, no SERS was observable (consistent with prior experiments, Figure 14 (a)), but following the $\mathrm{NaCl}$ spike, three vibrational modes were immediately observed to appear at 1333, 1405, and $1510 \mathrm{~cm}^{-1}$ Figure 14 (b). A second experiment (green) wherein $\mathrm{NaCl}$ was added before $\mathrm{R} 6 \mathrm{G}$ yielded no detectable SERS. This result implies that it is important for R6G to be adsorbed onto the Au@Ag prior to the aggregation event that triggers SERS, and this observation is completely consistent with the idea that interstitial sites are the crucial ones in generating intense SERS. When R6G is preadsorbed, the aggregation event is likely to trap a fraction of the molecules into interstitial sites which would likely be more inaccessible to diffusing R6G encountering pre-existing aggregates presenting a peripheral surface area to which it might adsorb without the proposed SERS enhancement. 

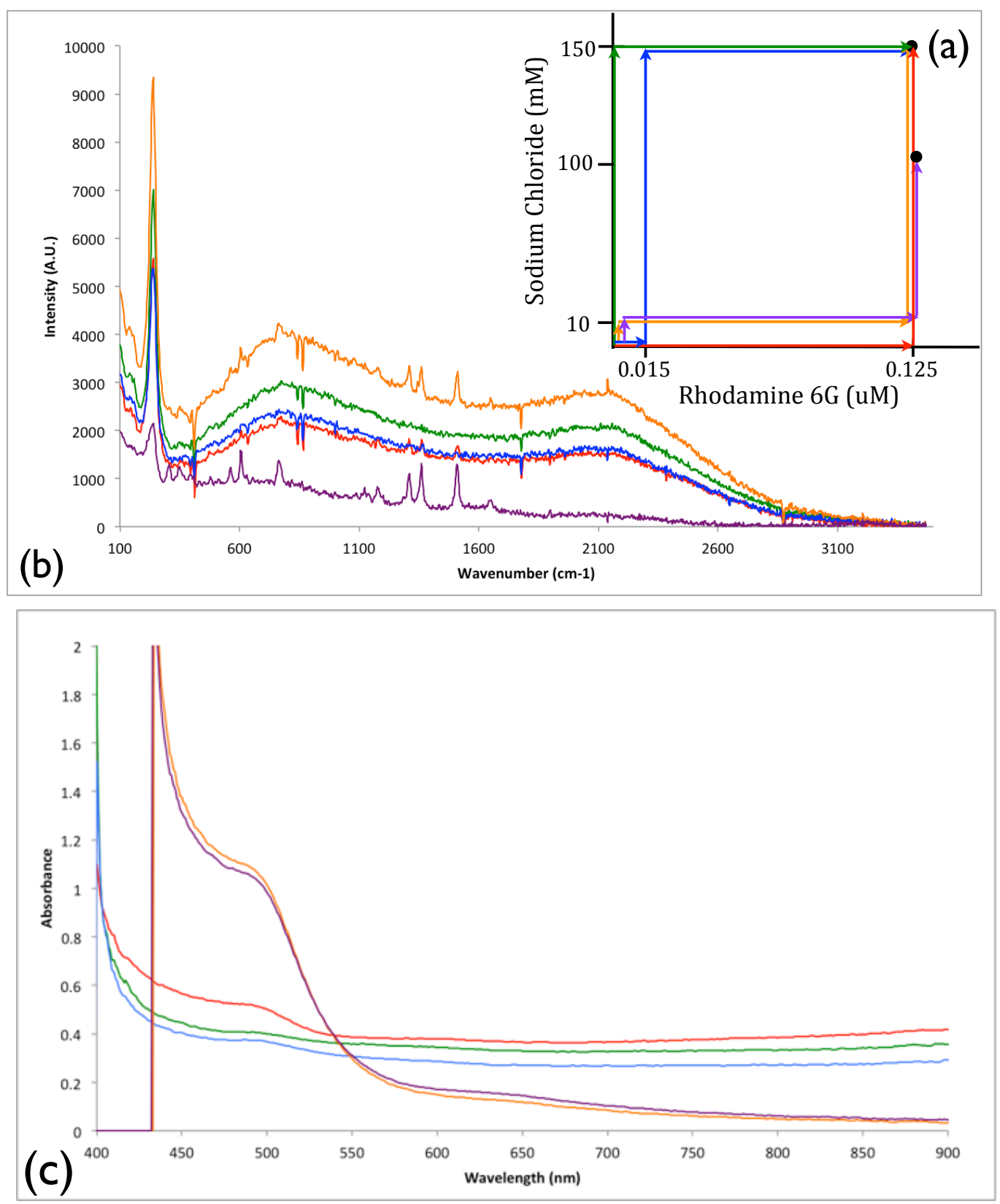

Figure 14: (a) is a diagram that illustrates both the order of addition and the final concentrations of $\mathrm{R} 6 \mathrm{G}$ and $\mathrm{NaCl}$ that are spiked into the $\mathrm{Au} @ \mathrm{Ag}$ solutions. Plots of Raman spectra (b) and visible extinction spectra (c) of each endpoint $(\bullet)$ indicated in the diagram are below. The colors of the plots correlate to the colors of the arrows in the 
diagram. Note that the downward spikes correspond to dark correction errors (improper subtraction of ambient light pollution) and are not related to Raman phenomena.

Having established that as little as $0.125 \mu \mathbf{M}$ R6G can be detected by preadsorption and aggregation of particles, three additional experiments were implemented as illustrated in Figure 14. Figure 14 (a) illustrates, using pairs or sets of arrows, the order and magnitudes of addition of $\mathrm{R} 6 \mathrm{G}$ and $\mathrm{NaCl}$ into the system. The experimental results can also be summarized in Table 2 below.

Table 2: Legend of details pertaining to Figure 14 Vis-NIR and Raman spectra. Spectra of indicated color were derived from Au@Ag solutions with the specified sequence of spikes, and yielding the indicated concentrations.

\begin{tabular}{|c|c|c|c|c|c|c|c|}
\hline \multirow{2}{*}{$\begin{array}{c}\text { Vis-NIR \& } \\
\text { SERS Spectra }\end{array}$} & \multicolumn{5}{|c|}{ Addition Order } & \multicolumn{2}{|c|}{ Raman Intensity } \\
\hline & & First & Second & Third & & R6G SERS & AgCl SERS \\
\hline 1 (red) & 0.125 & $\mu_{\mathrm{M}}$ R6G & $150 \mathrm{mM} \mathrm{NaCl}$ & - & - & Medium & Strong \\
\hline 2 (green) & 150 & $\mathrm{mM} \mathrm{NaCl}$ & $0.125 \mu_{\mathbf{M}} \mathbf{R 6 G}$ & - & - & Weak & Strong \\
\hline 3 (blue) & 0.015 & $\mu_{M}$ R6G & $150 \mathrm{mM} \mathrm{NaCl}$ & $0.125 \mu_{\mathbf{M}}$ & R6G & - & Strong \\
\hline 4 (orange) & 10 & $\mathrm{mM} \mathrm{NaCl}$ & $0.125 \mu_{\mathbf{M}} \mathbf{R} 6 \mathrm{G}$ & $150 \mathrm{mM}$ & $\mathrm{NaCl}$ & Strong & V.Strong \\
\hline 5 (purple) & 10 & $\mathrm{~m} \mathbf{M ~ N a C l}$ & $0.125 \mu_{\mathbf{M}} \mathbf{R} 6 \mathrm{G}$ & $100 \mathrm{mM}$ & $\mathrm{NaCl}$ & V.Strong & Weak \\
\hline
\end{tabular}

The first and second experiments are illustrated here with the red and green vectors respectively, which arrive at the same endpoint of $0.125 \mu \mathrm{M} \mathrm{R} 6 \mathrm{G} / 150 \mathrm{mM}$ 
$\mathrm{NaCl}$. In the second set of experiments, which arrive at the same final point, the blue vectors indicate that $0.015 \mu \mathrm{M}$ of $\mathrm{R} 6 \mathrm{G}$ was added first, followed by $150 \mathrm{mM} \mathrm{NaCl}$ and finally the concentration of R6G was brought up to $0.125 \mu \mathrm{M}$. This path allowed for an even smaller amount of R6G to preadsorb to the nanoparticles before executing the sequence identical to run 2, which yielded weak R6G SERS. Oddly, R6G SERS was not observed in this case. Clearly, the zero or low $[\mathrm{R} 6 \mathrm{G}]$ initial concentrations are negatively correlated with strong R6G SERS. It is entirely possible that the very low initial concentration $[0.015 \mu \mathrm{M}$ at pre-adsorption time] was well below the half-saturation (Langmuir $K_{D}$ value) level for $\mathrm{R} 6 \mathrm{G}$ at this surface, which is likely near $1 \mu \mathrm{M}^{50}$, which based on the expected linear relationship between coverage and concentration in this subsaturation range, would place the intensity below the instrumental detection limit for our system.

The last two experiments (4, orange and 5, purple) each begin with the addition of a significantly lower concentration of $\mathrm{NaCl}, 10 \mathrm{mM}$. This is followed by bringing the [R6G] to $0.125 \mu \mathrm{M}$, and finished with $[\mathrm{NaCl}]$ at either 100 or $150 \mathrm{mM}$. These sequences yielded the most intense SERS activity. Interestingly, the run concluding in $100 \mathrm{mM}$ $\mathrm{NaCl}$ appears to have produced both the strongest R6G SERS and the weakest $\mathrm{AgCl}$ SERS (band near $150 \mathrm{~cm}^{-1}$, vide infra). Evidently, $[\mathrm{NaCl}]$ final concentrations $>100 \mathrm{mM}$ may not be beneficial, but addition of low $[\mathrm{NaCl}]$ prior to adsorbing label may in fact be beneficial. 
The above may be correlated to the apparent states of aggregation by inspection of the Vis-NIR spectra in Figure 14 (c). Runs 1, 2, and 3 exhibited a dramatically broadened extinction profile indicative of extended aggregation. In these cases, $150 \mathrm{mM}$ $\mathrm{NaCl}$ was added in either the first (Run 2) or second $(1,3)$ step. For those cases where the sequence was $[\mathrm{NaCl}]=10 \mathrm{mM},[\mathrm{R} 6 \mathrm{G}]=0.125 \mu \mathrm{M},[\mathrm{NaCl}]>100 \mathrm{mM}$ (runs 4,5), the transverse dipolar plasmon band near $505 \mathrm{~nm}$ was well preserved, alongside a weak longitudinal dipolar band near $650 \mathrm{~nm}$, and without the extended broadband extinction; a signature consistent with either low aspect ratio rods, or dimeric particle pairs. ${ }^{48,51,52}$ This suggests that a. extended aggregation does not result from this protocol and $\mathbf{b}$. NP dimers may be promoted to some extent. The fact that these spectral features correlate most strongly with intense R6G SERS implies an important role for dimeric species in these solution phase systems, and that intense SERS is actually negatively correlated with the formation of extended aggregates.

Figure 15 shows the DLVO model prediction of interparticle potential energy versus interparticle distance for the three different $\mathrm{NaCl}$ concentrations used in these experiements. The $\zeta$-potentials measured for similarly sized $\mathrm{Ag}$ particles is used as an estimate of that expected for Au@Ag. Inspection of the plots reveals a significant (18 $\mathrm{kT})$ barrier to contact in the $10 \mathrm{mM}$ case, a modest one $(1.5 \mathrm{kT})$ at $100 \mathrm{mM}$ and a purely attractive interaction at $150 \mathrm{mM} \mathrm{NaCl}$, implying that in this latter case, irreversible aggregration would be expected to take place. 

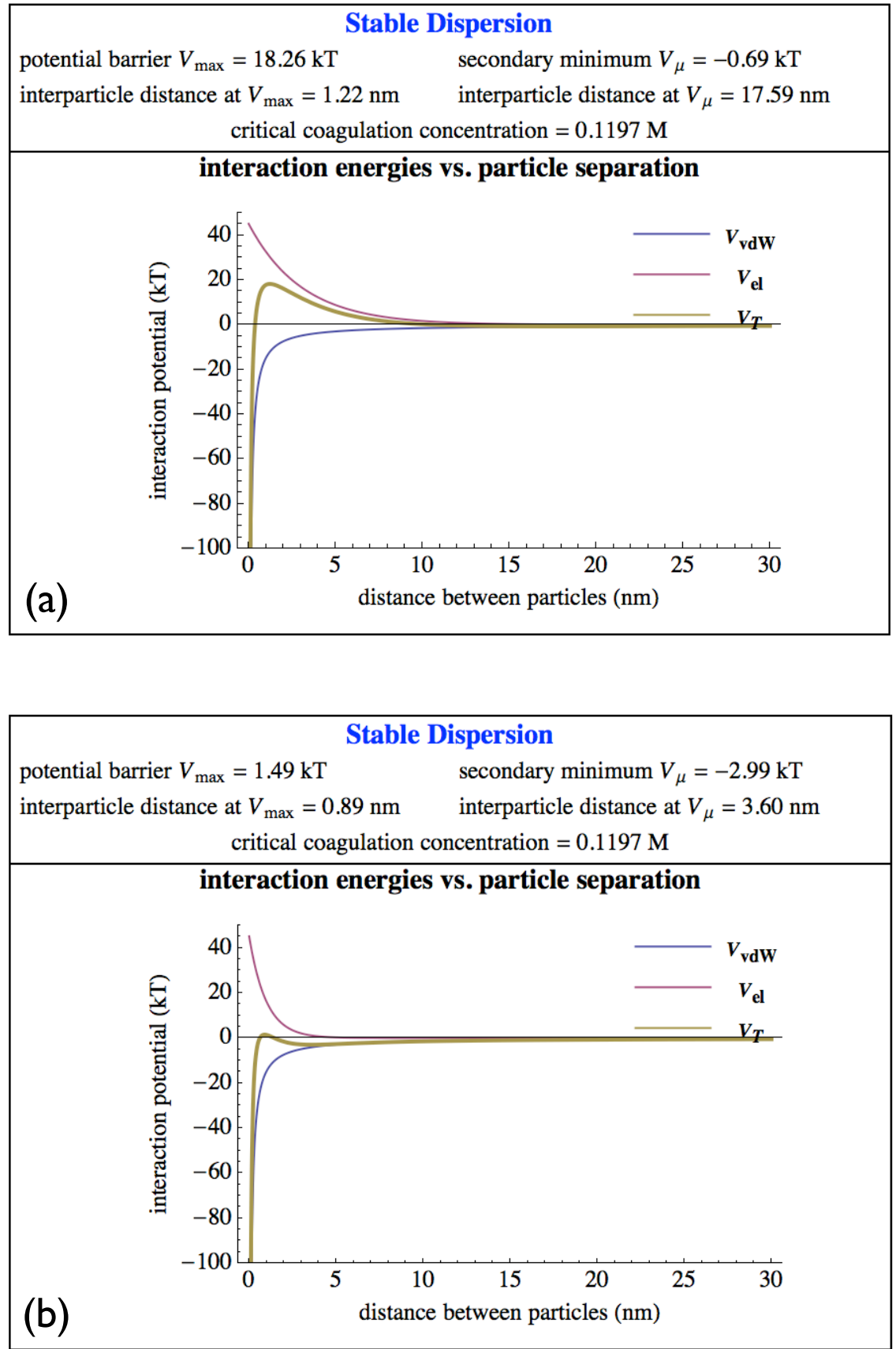


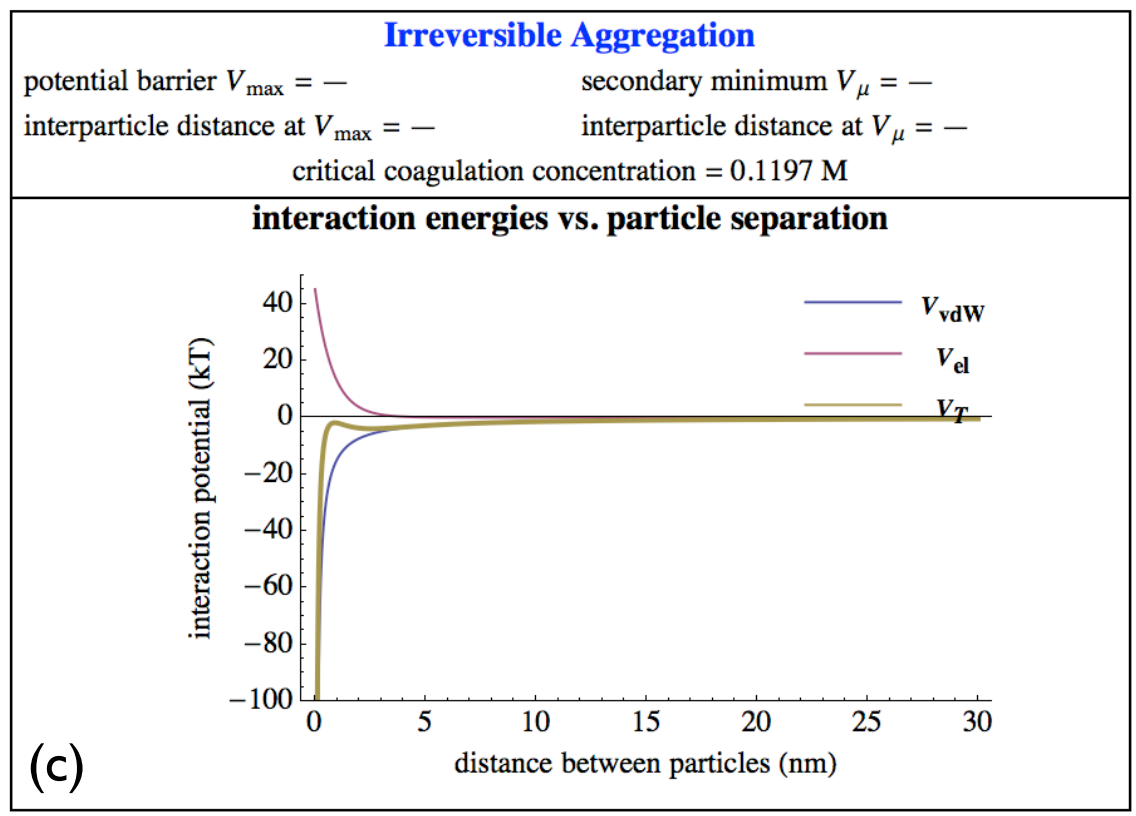

Figure 15: DVLO simulations for Ag nanoparticles coated with citrate ions with a radius of $20 \mathrm{~nm}$ at $25^{\circ} \mathrm{C}$. (a) uses a concentration of $10 \mathrm{mM} \mathrm{NaCl}$, while (b) and (c) is $100 \mathrm{mM}$ and $150 \mathrm{mM}$ respectively. The Mathematica source code for the models was adapted from Blaustein and Chappell. ${ }^{53}$

\section{NaCl Enhancement}

Figure 16 illustrates that a very substantial SERS signal was obtained after the addition of just $0.125 \mathrm{mM} \mathrm{R6G}$ and $10 \mathrm{mM} \mathrm{NaCl}$, conditions not expected to drive aggregation, it is suggested that the presence of $\mathrm{NaCl}$ alone can amplify the SERS in this system. Similar observations have been made by others ${ }^{54}$, but the nature as to the enhancement properties of $\mathrm{NaCl}$ are not entirely known. Generic ideas in this realm include the possibility that $\mathrm{NaCl}$ may somehow promote the adsorption of $\mathrm{R} 6 \mathrm{G}$ to $\mathrm{Ag}$ or that it may drive a re-orientation or R6G at the Ag surface which improves its Raman activity. ${ }^{54}$ Figure 17 show the SERS spectrum of R6G at Au@Ag with and without 
$\mathrm{NaCl}$ added to the solution. It is clearly evident that $\mathrm{NaCl}$ does not produce any significant spectral shift, nor a substantial re-distribution of mode intensities which might be expected to accompany a change in orientation. Therefore, a limiting case is assumed wherein $\mathrm{NaCl}$ promoted orientation changes increase the fraction of a unique SERS active orientation (i.e. other orientations are silent) then a re-orientation seems unlikely. Note that the mode at $236 \mathrm{~cm}^{-1}$ is associated with $\mathrm{NaCl}$ and is independent of the presence of R6G.

Both spectra have nearly identical vibration modes at the same wavenumber, except for the unique peak at $236 \mathrm{~cm}^{-1}$. Since there is no significant shift in any of the vibrational modes, strutuctural change to $\mathrm{R} 6 \mathrm{G}$ is not supported by this data.

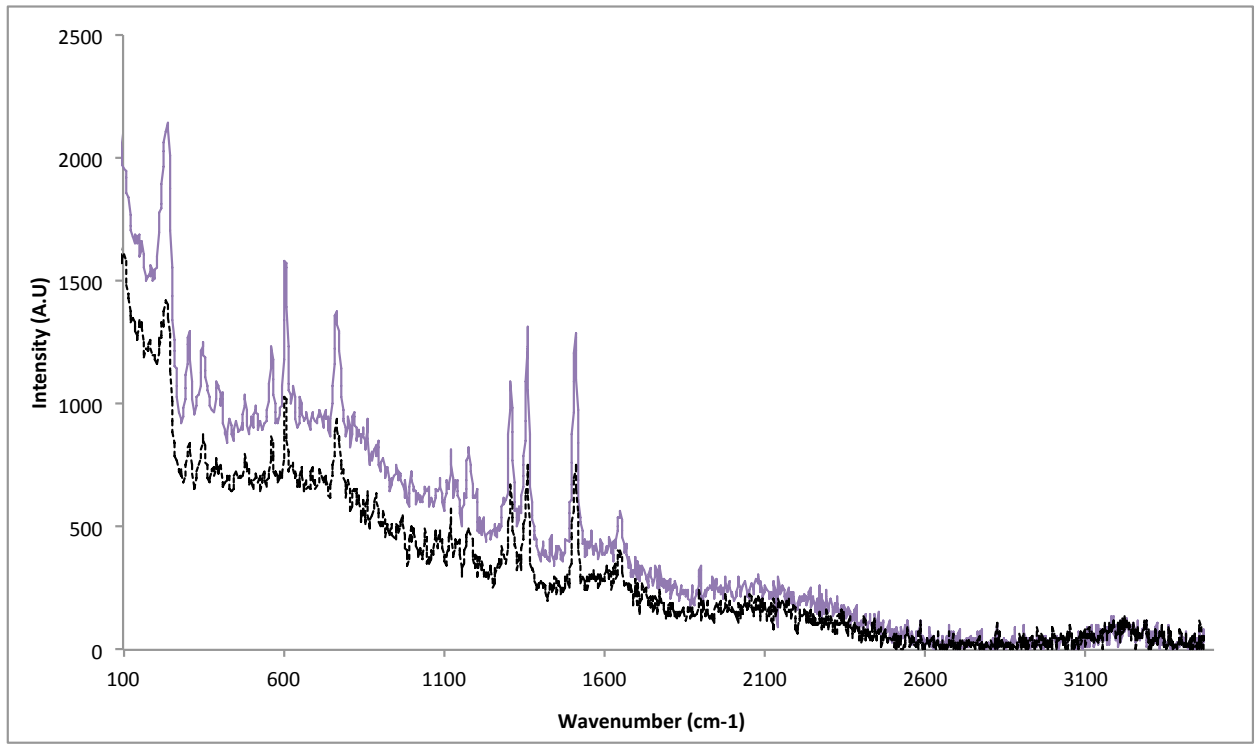

Figure 16: The SERS spectra of $0.125 \mathrm{mM} \mathrm{R6G}$ before (black) and after (purple) the $\mathrm{NaCl}$ concentration was increased to $100 \mathrm{mM}$ from $10 \mathrm{mM}$ 


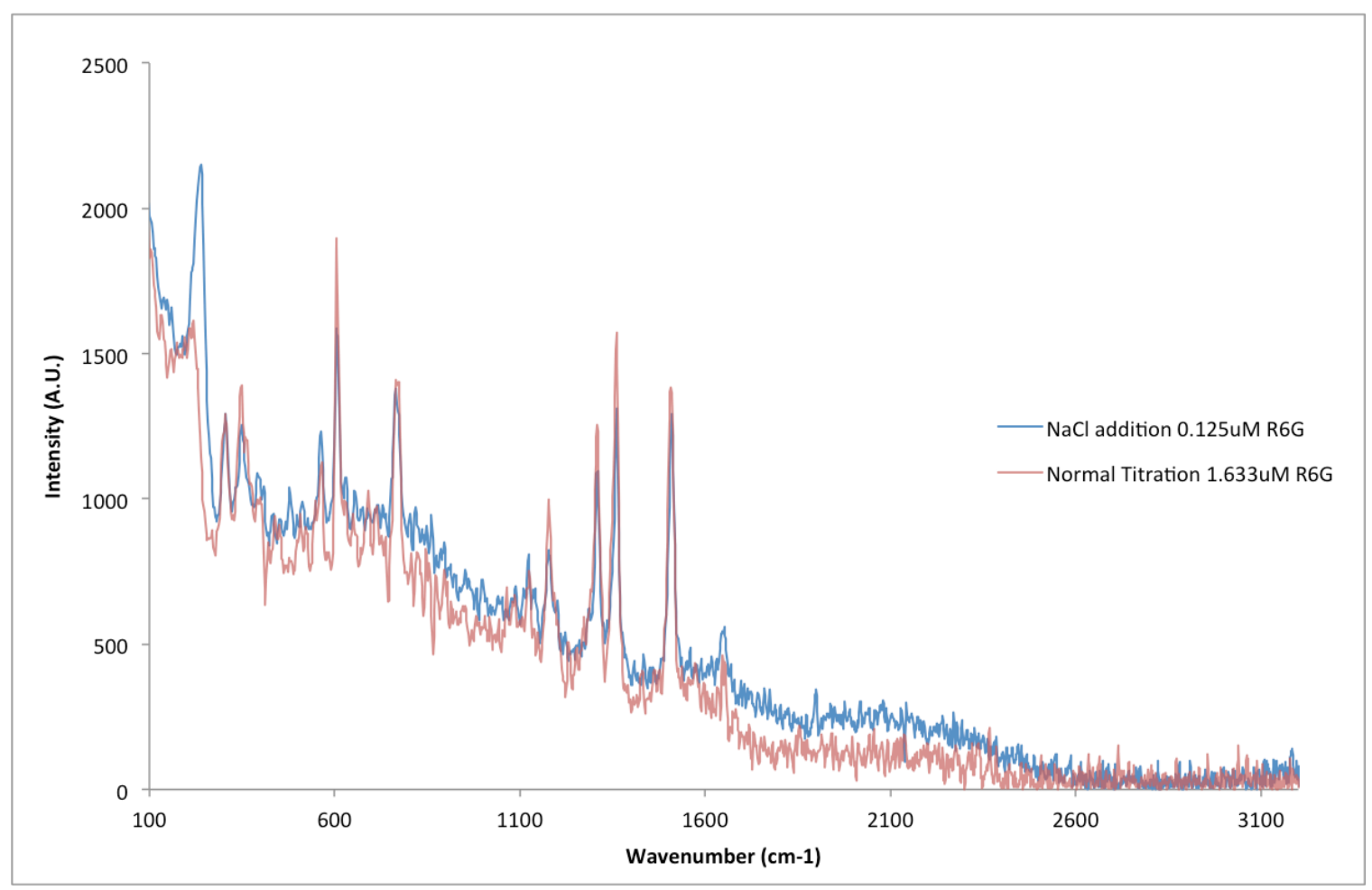

Figure 17: shows that the SERS spectrum of R6G has nearly identical Raman shifts as the SERS spectrum of R6G with the addition of $\mathrm{NaCl}$.

However the $236 \mathrm{~cm}^{-1}$ band was earlier attributed to a putative Ag-N (R6G) bond by Hildebrandt et.al ${ }^{50}$, who show it in systems using the alternate electrolytes $\mathrm{NaBr}$ and NaF. But these data simply conflict with ours as the above band is observed in our experiments in the absence of R6G, as illustrated in Figure 18. Therefore it is suggested that this is, in fact, an Ag-Cl stretching mode, and that perhaps the above measurements were conflicted by the presence of chloride from the original tetrachloroaurate precursors or the $\mathrm{HCl}$ cleaning protocols. 


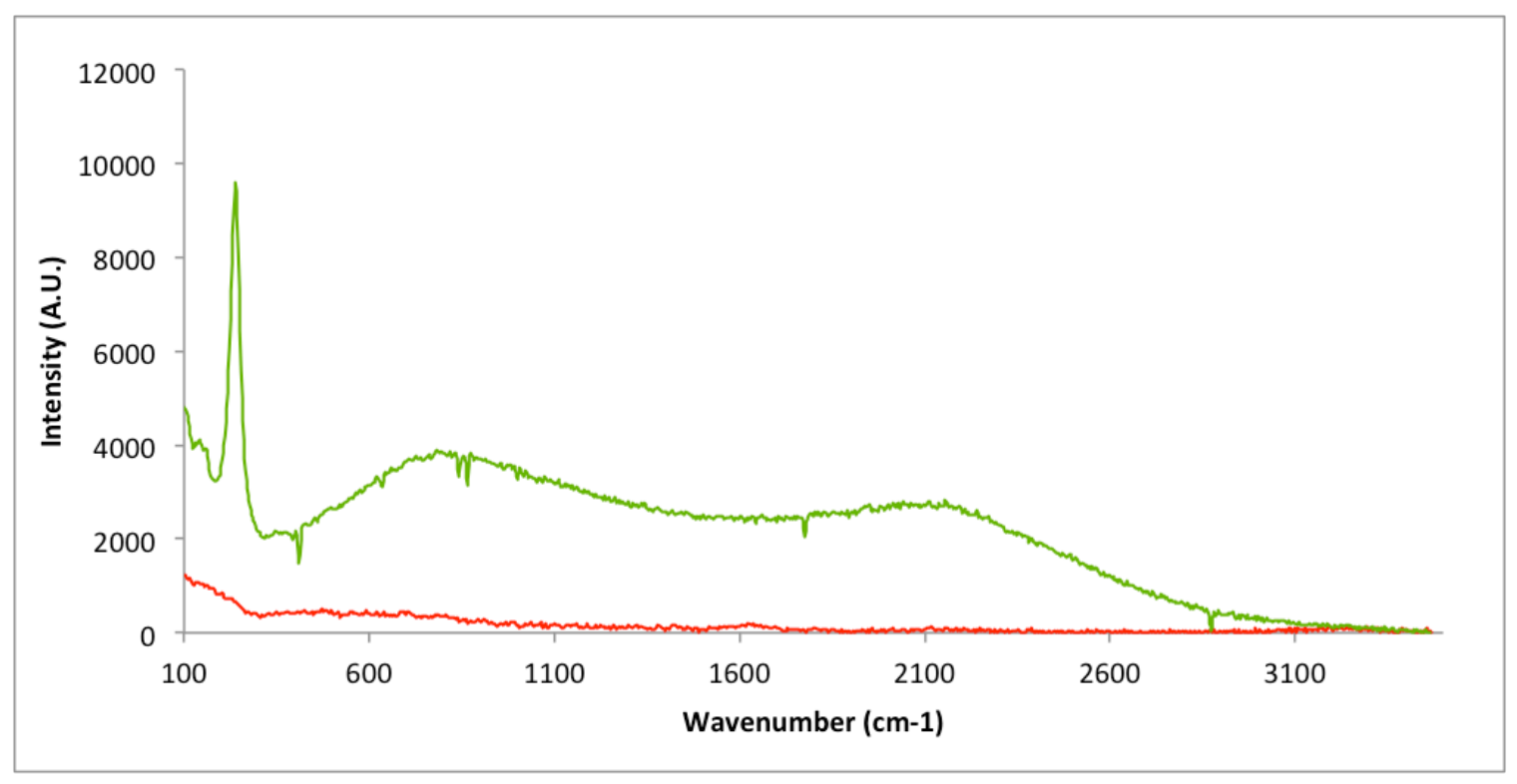

Figure 18: Raman spectrum of $\mathrm{Au} @ \mathrm{Ag}$ (red) and following addition of $150 \mathrm{mM} \mathrm{NaCl}$ (green).

Regardless of the exact origins of the $236 \mathrm{~cm}^{-1}$ peak, it is still likely that chloride is promoting the adsorption of $\mathrm{R} 6 \mathrm{G}$ in some way, e.g. via interaction with $\mathrm{AgCl}$ or $\mathrm{AgCl}_{2}{ }^{-}$ moieties on the nanoparticle surface. However, large $\mathrm{NaCl}$ concentrations, i.e. those that drive extended aggregation, do not benefit the SERS phenomenon. Figure 14b illustrates the essentially negative effect of crossing the aggregative threshold of $100-150 \mathrm{mM} \mathrm{NaCl}$. SERS intensities drop and the broad background signals (possibly 2-photon fluorescence from R6g) increases significantly. 
<smiles>CCN=c1cc2oc3cc(NCC)c(C)cc3c(-c3ccccc3C(=O)OCC)c-2cc1C</smiles>

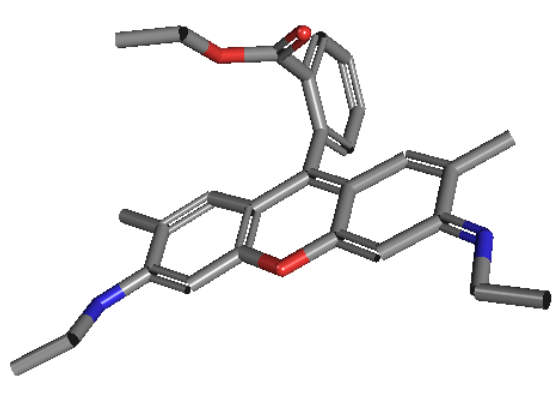

Figure 19: Chemical and 3D representation of $R 6 G$

Related reports have approached the subject of R6G molecular aggregates that may form on the surface of nanoparticles (both $\mathrm{H}$ and $\mathrm{J}$ types), and their potential to drive nanoparticle-nanoparticle interactions via intermolecular forces. ${ }^{55}$ It is reasoned that R6G molecules on separate nanoparticles are likely to ease the formation of nanoparticle dimers through formation of $\mathrm{H}$ or J-type dimeriation. The structure of R6G is given in Figure 19. Clearly, a variety of interactions are possible - stacking of the xanthine rings and hydrogen bonding (via $\mathrm{N}$ or $\mathrm{O}$ ) at various points. 


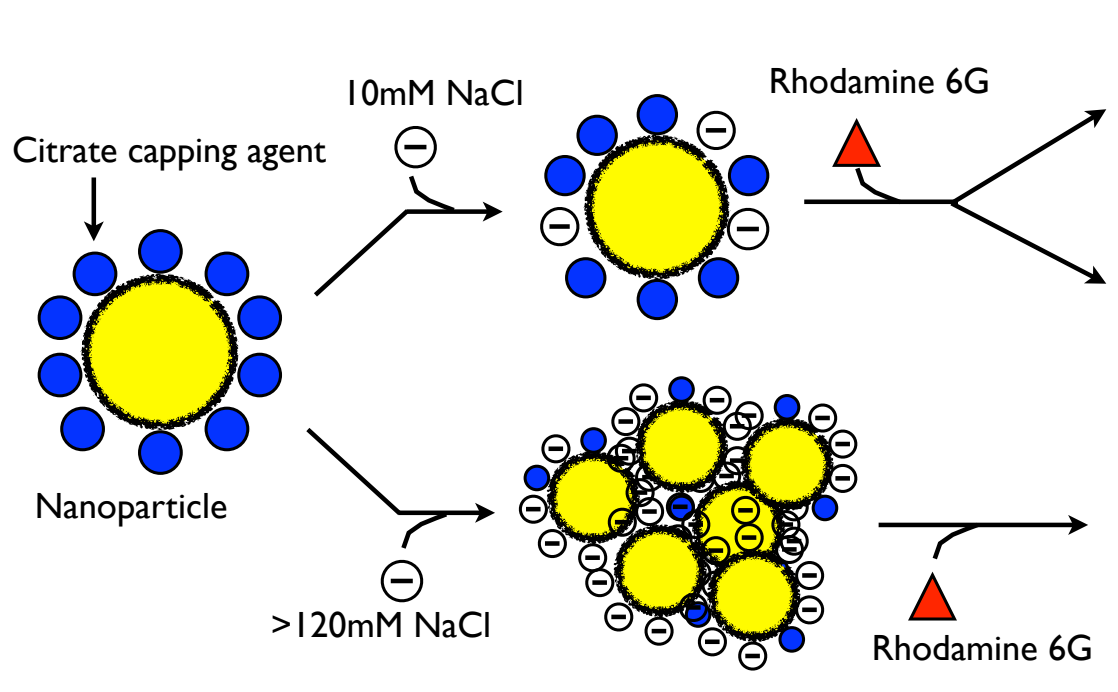

(d)
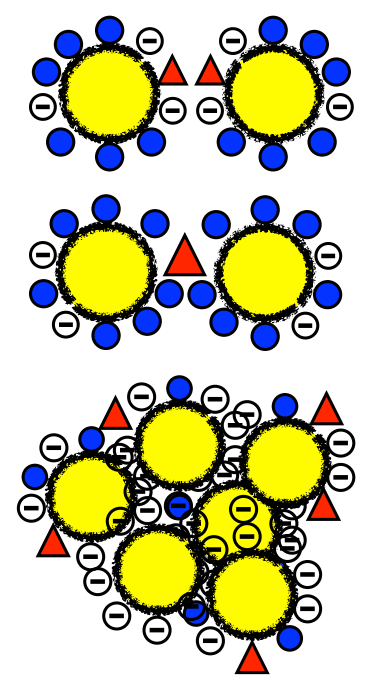

(e)

Scheme 1: Schematic that demonstrates the effects of addition of $\mathrm{NaCl}$ into the nanoparticle solution. (a) Shows the nanoparticle with the citrate ion-capping agents that provide the surface potential of the nanoparticles. (b) $\mathrm{NaCl}<50 \mathrm{mM}$ is added to the solution, the $\mathrm{Cl}^{-}$ions will displace some of the citrate ions around the nanoparticles. (c) R6G is added to the solution and causes the nanoparticles to flocculate by linking through formation of $\mathrm{H}$ or J-type dimers or bridging inter-nanoparticles into dimers and trimers. (d) $\mathrm{NaCl}>120 \mathrm{mM}$ is added to the nanoparticle solution, and cause the nanoparticles to coagulate. (e) Addition of R6G to coagulated nanoparticles does not allow the R6G to migrate into the SERS "hot spots."

All of the above observations are consistent with a schematic view of nanoparticle interactions leading to R6G SERS in the $\mathrm{NaCl}$ promoted $\mathrm{Au} @ \mathrm{Ag}$ system, which is illustrated above in Scheme 1. When low concentrations of $\mathrm{NaCl}$ are added to the nanoparticle solution, $\mathrm{Cl}^{-}$ions will associate with the NP surface, possibly displacing 
some of the citrate ions (b). These particles can remain in solution. When R6G is added to this solution, the R6G molescules will absorb to the nanoparticles surface. The R6G molecules will then further reduce the replusive force between NPs and promote dimerization or other limited aggregation as they are cationic at $\mathrm{pH} 7$ and NPs are anionic (c). ${ }^{56}$ Subsequent addition of large concentrations of $\mathrm{NaCl}$ can cause the entrapment of R6G molecules in the junctions between nanoparticles, and it is this junction or "hot spot" that will produce the largest SERS signal. On the other hand, if the initial introduction of $\mathrm{NaCl}$ is at too high of a concentration, i.e. above the cirtical coagulation concentraion of about $120 \mathrm{mM}$ aggregation will happen before R6G is introduced. In this case, when R6G is added to this solution, it will primarily occupy the surfaces of the aggregates and not benefit from the electromagnetic environment at NP-NP juctions, now occluded within larger structures, and SERS will not be observed.

\section{Conclusion}

By utilizing the combination of Raman and Vis-NIR spectroscopy, the origin of intense SERS was investigated with Au@Ag nanoparticles and R6G. Through a series of titrations of R6G into a solution of Au@Ag nanoparticles, it was concluded that SERS activity is correlated to the formation of nanoparticle aggregates since the latter present a clear signature in the VIS-NIR spectra, which tightly correlates to SERS activity. To clarify if aggregation was indispensible for SERS activity, the nanoparticles were aggregated using the SERS silent electrolyte $\mathrm{NaCl}$. By this method, SERS of R6G was recorded at a ten-fold lower concentration than was previously possible in this setting. 
This lends credence to the idea that in this case intense SERS is a function of nanoparticle aggregation and the entrapment of the SERS label within electromagnetic "hot spots" in the nanoscopic gaps between particles. These observations also underscore the interesting benefits that can be derived from the use of low and then higher concentrations of $\mathrm{NaCl}$ as a SERS promoting agent. When a SERS label is added before or with low $\mathrm{NaCl}$ concentration and then the $\mathrm{NaCl}$ concentration is elevated, below the point of flocculation, SERS is strongly promoted without inducing the sometimestroublesome broadband background emissions typical of these systems. 


\section{References}

(1) Raman, C. V. Journal of Physics 2 1928, 387-3.

(2) Hecht, E. In Optics; Addison-Wesley: Reading, Mass., 2002, pp 698.

(3) Smith, E.; Dent, G. In Modern Raman Spectroscopy: a practical approach; Wiley: Hoboken, NJ, 2005;

(4) Skoog, D.; Holler, F.; Crouch, S. Principles of Instrumental Analysis Sixth Edition; 6th ed.; Thomson, 2007, pp 481.

(5) Fleischmann, M.; Hendra, P. J.; McQuillam, A. J. Chemical Physics Letters 1974, 26, 163-166.

(6) Jeanmaire, David L.; Van Duyne, R. P.;. The Journal of Electroanalytical Chemistry and Interfacial Electrochemistry 1977, 84, 1-20.

(7) King, F. W.; Van Duyne, R. P.; Schatz, G. C. The Journal of Chemical Physics 1978, 69, 4472 .

(8) Laor, U.; Schatz, G. C. Chem. Phys. Lett. 1981, 82, 56.

(9) DiLella, D. P.; Gohin, A.; Lipson, R. H.; McBreen, P.; Moskovits, M. J. Chem. Phys. 1980, 73, 4282.

(10) Otto,A.;Timper,J.;Billmann,J.;Kovacs,G.;Pockrand,I.Surf. Sci. 1980, 92, L55.

(11) Van Duyne, R. P. Chemical and Biochemical Applications of Lasers; Moore, B. C., Ed.; Acedemic Press, Inc.: Burlington, MA, 1979; Vol. 4, pp 101-184.

(12) Nordlander, P.; Oubre, C.; Prodan, E.; Li, K.; Stockman, M. I. Nano Letters 2004, 4, 899-903.

(13) Schatz, G. C. Acc. Chem. Res. 1984, 17, 370.

(14) McCall, S.; Platzman, P.; Wolff, P. Phys. Lett. A 1980, 77, 381.

(15) Zeman, E. J.; Schatz, G. C. J. Phys. Chem. 1987, 91, 634.

(16) Lombardi, J. R.; Birke, R. L.; Lu, T.; Xu, J. J. Chem. Phys. 1986, 84, 4174.

(17) Adrian, F. J. J. Chem. Phys. 1982, 77, 5302. 
(18) Otto, A. Anal. Chim. Acta. 1980, 6, 309

(19) Morton, S. M.; Silverstein, D. W.; Jensen, L. Chemical reviews 2011, 111, 3962 94.

(20) Furtak, T.; Roy, D. Surf. Sci. 1985, 158, 126-146.

(21) Jiang, X. D.; Campion, A. Chem. Phys. Lett. 1987, 140, 95.

(22) McFarland AD, Young MA, Dieringer JA, Van Duyne RP. 2005. J. Phys. Chem. B 109:11279-85.

(23) C.L. Haynes, R.P. Van Duyne, J. Phys. Chem. B 105 2001, 5599.

(24) M.A. El-Sayed, Acc. Chem. Res. 34 2001, 257.

(25) S. Link, M.A. El-Sayed, J. Phys. Chem. B 103 1999, 8410.

(26) U. Kreibig, M. Gartz, A. Hilger, H. Hovel, Optical Investigations of Surfaces and Interfaces of Metal Clusters, JAI Press, Stamford, CT, USA, 1998, p. 345

(27) Khlebtsov, N. G.; Dykman, L. a. Journal of Quantitative Spectroscopy and Radiative Transfer 2010, 111, 1-35.

(28) Wentong Lu, Sri Ranjini Arumugam, Dulal Senapati, Anant K. Singh, Tahir Arbneshi, Sadia Afrin Khan, Hongtao Yu, and P. C. R. ACS nano 2010, 4, 17391749.

(29) Wustholz, K. L.; Henry, A.-I.; McMahon, J. M.; Freeman, R. G.; Valley, N.; Piotti, M. E.; Natan, M. J.; Schatz, G. C.; Van Duyne, R. P. Journal of the American Chemical Society 2010, 132, 10903-10.

(30) Mie, G. Ann. Phys. Weinheim, Ger. 1908, 25, 377.

(31) Xie, W.; Su, L.; Shen, A.; Materny, A.; Hu, J. Journal of Raman Spectroscopy 2011, 42, 1248-1254.

(32) Mayer, K. M.; Hafner, J. H. Chemical reviews 2011, 111, 3828-57.

(33) Xu, W.; Xue, X.; Li, T.; Zeng, H.; Liu, X. Angewandte Chemie (International ed. in English) 2009, 48, 6849-52.

(34) Liu, B.; Han, G.; Zhang, Z.; Liu, R.; Jiang, C.; Wang, S.; Han, M. Anal. Chem. 2012, 84, 255-261. 
(35) Kimling, J.; Maier, M.; Okenve, B.; Kotaidis, V.; Ballot, H.; Plech, a The journal of physical chemistry. B 2006, 110, 15700-7.

(36) Lu, Y.; Liu, G. L.; Lee, L. P. Nano Letters 2005, 5, 5-9.

(37) Jain, P. K.; Lee, K. S.; El-Sayed, I. H.; El-Sayed, M. A. The Journal of Physical Chemistry B 2006, 110, 7238-7248.

(38) Cortie, M. B.; McDonagh, A. M. Chemical reviews 2011, 111, 3713-35.

(39) Mayer, K. M.; Hafner, J. H. Chem. Rev. 2011, 111, 3828-57.

(40) Thomas, K. G.; Kamat, P. V 2003, 36, 888-898.

(41) Wentong Lu, Sri Ranjini Arumugam, Dulal Senapati, Anant K. Singh, Tahir Arbneshi, Sadia Afrin Khan, Hongtao Yu, and P. C. R. ACS Nano 2010, 4, 1739 1749 .

(42) Hazarika, P.; Ceyhan, B.; Niemeyer, C. M. Angew. Chemie 2004, 116, 6631-6633.

(43) McMahon, J. M.; Li, S.; Ausman, L. K.; Schatz, G. C. The Journal of Physical Chemistry C 2012, 116, 1627-1637.

(44) McMahon, J.; Gray, S.; Schatz, G. Physical Review B 2011, 83, 1-5.

(45) Nordlander, P.; Oubre, C.; Prodan, E.; Li, K.; Stockman, M. I. Nano Letters 2004, 4, 899-903.

(46) Kim, T.; Lee, C.-H.; Joo, S.-W.; Lee, K. 2008, 318, 238-43.

(47) Vernon, K. C.; Funston, A. M.; Novo, C.; Gomez, D. E.; Mulvaney, P.; Davis, T. J. Nano Lett. 2010, 10, 2080-2086.

(48) Marinica, D. C.; Kazansky, a K.; Nordlander, P.; Aizpurua, J.; Borisov, a G. Nano letters 2012, 12, 1333-9.

(49) Funston, A. M.; Novo, C.; Davis, T. J.; Mulvaney, P. Geometries.Nano Lett 2009, $9,1651-1658$.

(50) Hildebrandt, P.; Stockburger, M. journal of physical Chemistry 1984, 88, 59355944.

(51) Dreaden, E. C.; Near, R. D.; Abdallah, T.; Talaat, M. H.; El-Sayed, M. A. Appl. Phys. Lett. 2011, 98, 183115-183115-3. 
(52) Jain, P. K.; Lee, K. S.; El-Sayed, I. H.; El-Sayed, M. A. The Journal of Physical Chemistry B 2006, 110, 7238-7248.

(53) Gail Blaustein and Mark Chappell "Interaction Energies between Spherical Colloidal Particles in a Symmetric Electrolyte Solution"; http://demonstrations.wolfram.com/InteractionEnergiesBetweenSphericalColloidal ParticlesInASymm/;Wolfram Demonstrations Project

(54) Otto, A.; Bruckbauer, A.; Chen, Y. J. Mol. Struct. 2003, 661, 501-514.

(55) Zhao, J.; Jensen, L.; Sung, J.; Zou, S.; Schatz, G. C.; Duyne, R. P. Van Journal of the American Chemical Society 2007, 129, 7647-56.

(56) Ilich, P.; Mishra, P.; Macura, S.; Burghardt, T. Spectrochimica Acta Part A: Molecular and Biomolecular Spectroscopy 1996, 52, 1323-1330. 